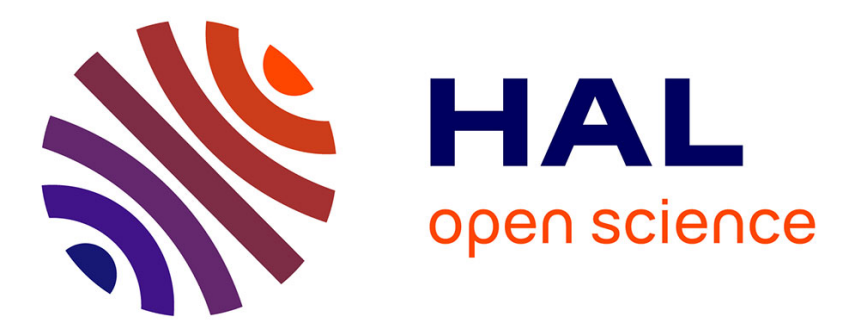

\title{
Pressure dependence of the electrical transport in granular materials
}

\author{
Mathieu Creyssels, C. Laroche, Eric Falcon, Bernard Castaing
}

\section{To cite this version:}

Mathieu Creyssels, C. Laroche, Eric Falcon, Bernard Castaing. Pressure dependence of the electrical transport in granular materials. European Physical Journal E: Soft matter and biological physics, 2017, 40 (5), pp.56. 10.1140/epje/i2017-11543-3 . hal-01724830

\section{HAL Id: hal-01724830 \\ https://hal.science/hal-01724830}

Submitted on 9 Apr 2019

HAL is a multi-disciplinary open access archive for the deposit and dissemination of scientific research documents, whether they are published or not. The documents may come from teaching and research institutions in France or abroad, or from public or private research centers.
L'archive ouverte pluridisciplinaire HAL, est destinée au dépôt et à la diffusion de documents scientifiques de niveau recherche, publiés ou non, émanant des établissements d'enseignement et de recherche français ou étrangers, des laboratoires publics ou privés. 


\title{
Pressure dependence of the electrical transport in granular materials
}

\author{
M. Creyssels ${ }^{1 \mathrm{a}}$, C. Laroche $^{2}$, E. Falcon ${ }^{2}$, and B. Castaing ${ }^{3}$ \\ 1 Laboratoire de Mécanique des Fluides et Acoustique, Ecole centrale de Lyon, CNRS, Univ. de Lyon, 69134 Ecully, \\ France \\ 2 Univ. Paris Diderot, Sorbonne Paris Cité, MSC, CNRS, 75013 Paris, France \\ 3 Laboratoire des Ecoulements Géophysiques et Industriels, Univ. Grenoble Alpes, CNRS, 38058 Grenoble, France
}

Received: date / Revised version: date

\begin{abstract}
We report on systematic measurements of the electrical resistance of one- and three-dimensional (1D and 3D) metallic and oxidized granular materials under uni-axial compression. Whatever the dimension of the packing, the resistance follows a power law versus the pressure $\left(R \propto P^{-\alpha}\right)$, with an exponent $\alpha$ much larger than the ones expected either with elastic or plastic contact between the grains. A simple model based on a statistical description of the micro-contacts between two grains is proposed. It shows that the strong dependence of the resistance on the pressure applied to the granular media is a consequence of large variabilities and heterogeneities present at the contact surface between two grains. Then, the effect of the three dimensional structure of the packing is investigated using a renormalization process. This allows to reconcile two extreme approaches of a 3D lattice of widely distributed resistances: the effective medium and the percolation theories.
\end{abstract}

PACS. 45.70.-n Granular systems - 72.80.-r Electrical conductivity of specific materials

\section{Introduction}

The unusual transport properties of granular matter can come from different sources. They can originate in the grain-grain contact properties [1], or in the three-dimensional structure of the random packing of grains $[2,3]$. In the case of electrical conductivity of metallic granular samples, we examine here, all the resistance is due to the thin oxide layers covering the grains. The properties of the graingrain contact are thus an essential ingredient. However, the current is known to circulate within preferred paths [4-6]. Mechanical stresses are also concentrated in granular matter along preferred paths [7]. Do the mechanical and electrical paths coincide or are the latter a simple percolation lattice due to the extreme variability of the grain-grain contact electrical resistance? At the end, is the global electrical resistance of a granular sample a sensitive probe of this microscopic structure?

In order to contribute to these questions, we examine here the pressure-dependent electrical resistance of one- and three-dimensional (1D and 3D) metallic and oxidized granular materials under uni-axial compression. The paper is organized as follows. After presenting the experimental apparatus and procedures (Sect. 3), we first show the power-law dependence of the resistance of powder samples with the force (Sect. 4.1), then the reproducibility of the measurements, in terms of the effective

\footnotetext{
${ }^{a}$ e-mail: mathieu.creyssels@ec-lyon.fr
}

resistivity of an effective medium (Sect. 4.2), and finally we demonstrate that the resistance of a chain of beads also exhibits a power-law behaviour with the applied force (Sect. 4.3). In section 5, we propose a model for explaining this power-law dependence of the resistivity on the applied force whatever the dimension of the granular packing. The last section (6) is dedicated to the main observed difference between the $1 \mathrm{D}$ and $3 \mathrm{D}$ experimental results that is the reproducibility of the measurements. We discuss its physical origin and relates it to the effective medium and the percolation theories.

\section{State of the art}

The mechanical behaviour of two spherical grains in direct contact is rather well known. Indeed, for small static forces, the Hertz law describes remarkably well the nonlinear interaction between two elastic spheres. It predicts that the contact area $(A)$ as a function of the force exerted on the spheres $(f)$ follows a power-law behaviour [8]:

$$
A \sim f^{2 / 3} .
$$

The force divided by the contact area defines the contact pressure $p$. Then, in the elastic regime (ER), $p \sim f^{1 / 3}$. At higher forces, when the pressure exceeds the yield strength of the material, a permanent plastic deformation occurs at 


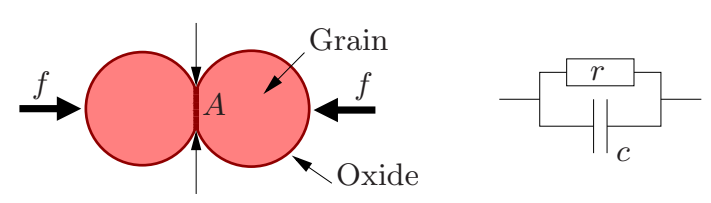

Fig. 1. Left: Two metallic grains under compression. $A$ is the contact area. An insulating film composed of oxides or impurities covers each grain. Right: Electrical equivalent circuit of the contact between the grains. $r$ and $c$ are respectively the resistance and the capacitance at low frequency (see [6]).

the contact and the previous power law becomes [8]:

$$
A=f / Y \text {. }
$$

In this plastic regime (PR), the contact pressure is almost constant and approximately equal to the yield stress $(Y)$ whatever the force applied to the grains.

Contrary to the mechanical behaviour, the force-dependent electrical resistance of two metallic grains in contact is still an open problem. For the special case of clean and non-oxidized surface contact, experimental and theoretical studies showed that the electrical resistance thus measured is proportional to the inverse of the cube root of the force when the contact pressure is lower than the plastic yield stress and to the inverse of the square root of the force in the plastic regime $[1,9,10]$. Indeed, the resistance of the metallic contact $(r)$ is a result of the constriction of the current stream through the small contact area $(A)$. It depends only on the resistivity of the metal $\left(\rho_{m}\right)$ and on the radius of the contact area: $r \sim \rho_{m} A^{-1 / 2}$. For elastic spheres, from Eq. (1) we get $r \sim f^{-1 / 3}$ whereas Eq. (2) (PR) leads to $r \sim f^{-1 / 2}$. However, in realistic granular packings, grains are not clean and covered by an oxide layer or another contaminant film which resistivity is largely greater than the metal one (Fig. 1). In that case, both the bulk of the metallic grain and the constriction resistances are lower than the resistance of the insulating layer. Assuming the Ohmic law is valid and the current passes uniformly through the oxide film, the contact resistance can be written as

$$
r=\rho_{o x} \delta_{o x} / A,
$$

where $\rho_{o x}$ and $\delta_{o x}$ are respectively the oxide resistivity and its thickness. Using equations (1) or (2), this very simple model of the electric contact predicts a power-law behaviour of the force-dependent resistance:

$$
\begin{array}{ll}
r & \sim f^{-2 / 3} \quad(\mathrm{ER}), \\
r & \sim f^{-1} \quad(\mathrm{PR}) .
\end{array}
$$

This theoretical scaling has not yet been observed in a compressed packing of metallic grains. Some experimental studies observed a power-law dependence of the resistance on the force $\left(r \sim f^{-\alpha}\right)$ but with an exponent $\alpha$ greater than one $[3,11,12]$. For several granular materials, this exponent varies between 2 and 3 ! Up to now, no clear explanation has been given to interpret the large

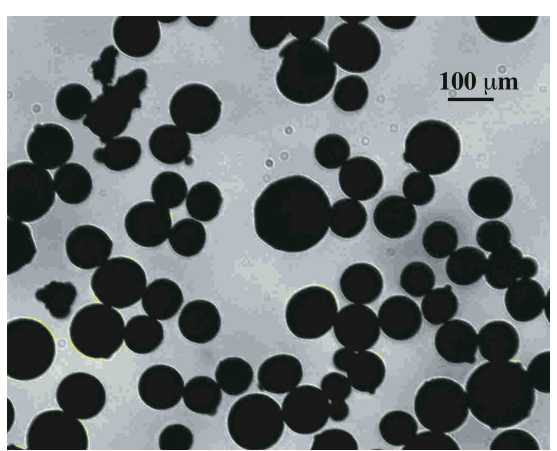

Fig. 2. Image of grains of copper powder through a microscope.

exponent observed in the experiments. In particular, exponents larger than 1 may come from the structural disorder observed for the distribution of contact forces within three-dimensional grain assemblies, but they can also be a characteristic feature of the electrical contact between two grains covered by oxides, independently of the structure of the force network in the granular medium. With the help of experiments done both on metallic powders and on chains of metallic beads, we show in this paper that the strong dependence of the electrical resistance on the force only comes from the electrical properties of the contact between two metallic grains.

\section{Experimental methods}

\subsection{Metallic powders (3D samples)}

The grains of our samples [13] are spherical copper particles, of average diameter $\langle d\rangle=80 \mu \mathrm{m}$. They are polydispersed, their diameters spanning a range of approximately a factor two. The powder we have used come from two different commercial batches called here "batch I" and "batch II", of same characteristics. We looked the grains from the two batches through a microscope (see Fig. 2) and we have not noticed any difference in the shape or in the polydispersity of the grains. Maybe more sophisticated analysis of the grains would reveal some physical or chemical differences between the two powders. However, for this present study, this tedious work is unnecessary.

The measured volumetric mass density is $\mu_{\text {eff }}=5.5$ $\mathrm{g} / \mathrm{cm}^{3}$. As Goodfellow ensures a purity in copper of the grains equal to or greater than $99 \%$ [13], the packing density of the samples we have used is $\mu_{e f f} / \mu_{C u}=62 \%$. This value is entirely consistent with the measurements of packing density of spheres in three dimensions given by several authors [14-16]. The number of grains per unit volume $(n)$ can also be calculated using the average diameter:

$$
n=\frac{\mu_{e f f}}{\mu_{C u}} \frac{6}{\pi\langle d\rangle^{3}}=2300 \text { grains } / \mathrm{mm}^{3} .
$$

The samples of powder are placed in various cylindrical containers, made of PMMA, and confined between two 


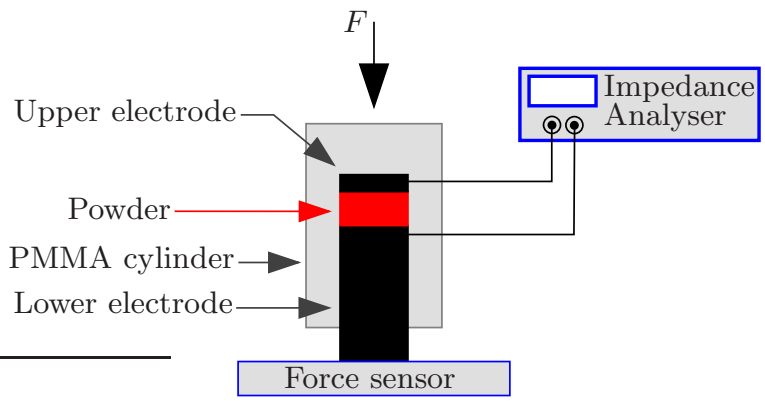

Fig. 3. Experimental set-up for the 3D samples [6].

brass electrodes (Fig. 3). A load is directly applied to the upper electrode and the electrical measurements are then performed only after waiting the relaxation of mechanical stress inside the powder. The force $(F)$ exerted on the electrodes is measured by a resistive force sensor (FGP Instrumentation $\left.^{\mathrm{TM}}\right)$. We call $\Phi$ the diameter of the container, $5 \leq \Phi \leq 15 \mathrm{~mm}$, and $h$ the distance between the electrodes $(1 \leq h \leq 18 \mathrm{~mm})$. After each measurement at a single applied force, we change the powder and the following sample is made of fresh and unused powder.

Due to variations in the grain sizes, non-regular arrangement of the grains and non-linear interaction between the grains, the distribution of stresses in the granular medium is both complex and inhomogeneous. Stresses are notably transmitted through the material along force chains involving only a fraction of all grains [17]. Hence, the average compression force $(\langle f\rangle)$ between two neighbour grains in contact cannot be known with a good precision. However, we can estimate it as the product of the macroscopic pressure applied to the powder $(P)$ with the surface area defined as $\langle s\rangle=n^{-2 / 3}$ :

$$
\langle f\rangle \approx P n^{-2 / 3}
$$

Indeed, for any horizontal cross-sectional area of the cylindrical container, the average number of grains cut by this surface is $\pi \Phi^{2} / 4 \times n^{2 / 3}$, hence $\langle s\rangle$ represents the surface area taken by one grain. As the minimum pressure applied to the powder is $1 \mathrm{~N} / \mathrm{mm}^{2}$, the average microscopic force is in that case $6 \mathrm{mN}$ using Eq. (7). It is already enough to deform plastically the grains. Indeed, the yield stress of copper is equal to $70 \mathrm{~N} / \mathrm{mm}^{2}$ and, in the plastic regime, the contact area is given by Eq. (2). So, when the force reaches $6 \mathrm{mN}$, the diameter of the contact area goes up to approximately $10 \mu \mathrm{m}$ and is greater than $\langle d\rangle / 10$. It is thus reasonable to think that a large number of grains in the packing endures irreversible plastic deformation. That is why the container is refilled with a new sample of powder before each experimental run.

The electrical resistance is measured using a Hewlett Packard 4192A impedance analyser. Notice that the amplitude of the alternative voltage applied to the powder sample is fixed to a low value $\left(U_{R M S}=100 \mathrm{mV}\right)$ in order to avoid any voltage-induced non-linear effect $[6,18]$. Besides, previous results [6] showed that the resistance is frequency independent at low frequencies $(\leq 1 \mathrm{kHz})$. In

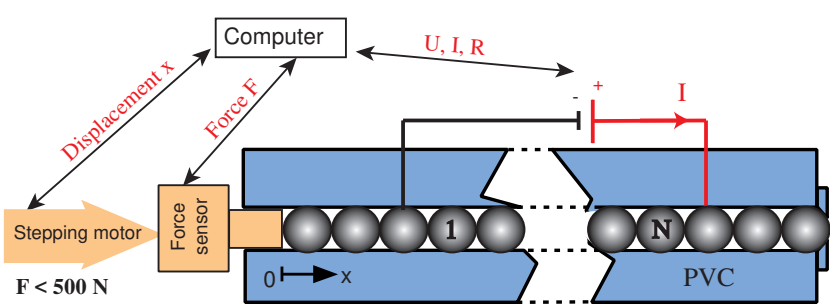

Fig. 4. The chain of stainless steel beads [19].

addition, the ac conductivity collapses to the dc conductivity when the frequency is low. The main interest to use an impedance analyser in place of a standard multi-meter is the possibility to measure both the amplitude and the phase of the electrical response of the powder.

\subsection{Metallic beads (1D samples)}

We also used one-dimensional samples [5,19] (Fig. 4). Here, the grains are $8 \mathrm{~mm}$ in diameter and stainless steel rolling beads. They are confined in a straight channel, and a force can be applied on this chain of beads by means of a piston in the range from 1 to $500 \mathrm{~N}$. The force is measured by a resistive force sensor (FGP Instrumentation ${ }^{\mathrm{TM}}$ ). The piston is moved by a stepper motor and then its displacement is controlled by a computer. Hence, moving the piston, the force can be easily changed and adjusted to the chosen value. Besides, the piston can be moved backward far from the first bead in order to have the possibility to roll the beads and to change the contacts between the beads. Note that a very small clearance of $2 / 100 \mathrm{~mm}$ is provided in the channel, so that the beads can move freely along the chain axis but not laterally. Hence, contrary to the powder, for the chain of beads, the same force is applied to each bead since the channel is straight and lateral displacements of the beads are negligible compared to axial displacements of the beads. Besides, [19] measured the total displacement of the chain and showed that the deformation of the beads is in the elastic regime [Hertz's law, Eq. (1)] whatever the force applied to the chain (see Fig. 2 in [19]). Unlike the experiments with powders for which a new sample is systematically used for each experimental run, the beads are not changed. But, to obtain reproducible measurements, the piston is moved backward and the beads are systematically rolled so that the contacts between the beads are thus systematically changed before each measurement.

For measuring the electrical resistance, two conductive leads are soldered on the first and last beads of the chain and they are connected to a direct voltage source (Keithley 2400) which also gives a measurement of the electric current. The measured resistance is always largely higher than both the resistance of the leads and the stainless steel bulk material. Besides, careful attention has been paid to inject to the chain of beads very low electric power, in order to avoid any decrease of the resistance due to the current flowing through the contacts between the beads. $[5]$ showed that the tension $(U)$ versus current $(I)$ charac- 
teristic of the chain of beads (but also for two- and threedimensional granular metals) exhibits three different and successive regimes when the current is increased. At very low currents, the $U-I$ characteristic is linear and constant in time showing that the resistance is independent of the injected current. Then, for higher currents, the $U-I$ characteristic becomes non-linear but stays reversible that is the $U-I$ characteristic is the same when the current is decreased after being increased. Finally, if the current exceeds a critical value, the resistance decreases irreversibly. This critical transition from an insulating to a conductive state for the granular metal is similar to the "Branly effect" $[5,19,20]$. For this present study, this transition is not studied and all the given resistances have been measured in the first regime for which the $U-I$ characteristic is linear and reversible. Besides, all the electrical devices are connected to the same computer in order to control and measure at the same time the mechanical force and the electrical resistance.

\section{Experimental results}

We present here the electrical resistivity measurements of metallic powders and of chains of beads under axial mechanical force. We also show that the powder behaves like an effective medium constituted by a very large number of grains $(>100000)$. But the experimental results on chains of beads indicate that the not obvious behaviour of the electrical resistance as a function of force comes from some complexities of the electrical contact between two grains rather than some rearrangements of the current conducting channels in the powder.

\subsection{Electrical properties of the powders}

We begin this section by giving our experimental results on the electrical conductivity of the metallic powder from batch I as a function of the applied mechanical force. In Fig. 5, the resistance $R$ of samples of different masses and sizes is plotted against the force. The logarithmic plot suggests that a power law

$$
R \propto F^{-\alpha_{p_{I}}}
$$

well fits the data. The best fit is obtained for $\alpha_{p_{I}}=1.7$ whatever the mass or the size of the sample. We want to emphasize both the reproducibility of the resistance for a given sample geometry and the large value of $\alpha_{p_{I}}$, which will be deeply discussed throughout this article. Indeed, $\alpha_{p_{I}}$ is greater than 1 and thus also $2 / 3$ that are the slopes predicted by Eqs. (5) and (4) respectively.

As explained in the section 3, low frequency ac measurements allow us to determine the effective capacitance $(C)$ of the powder sample, in parallel to the resistance. The dependence of $C$ on the applied force, for a sample of batch I, in the same conditions than for Fig. 5, is shown in Fig. 6. The capacitance is clearly proportional to the applied force:

$$
C \propto F
$$

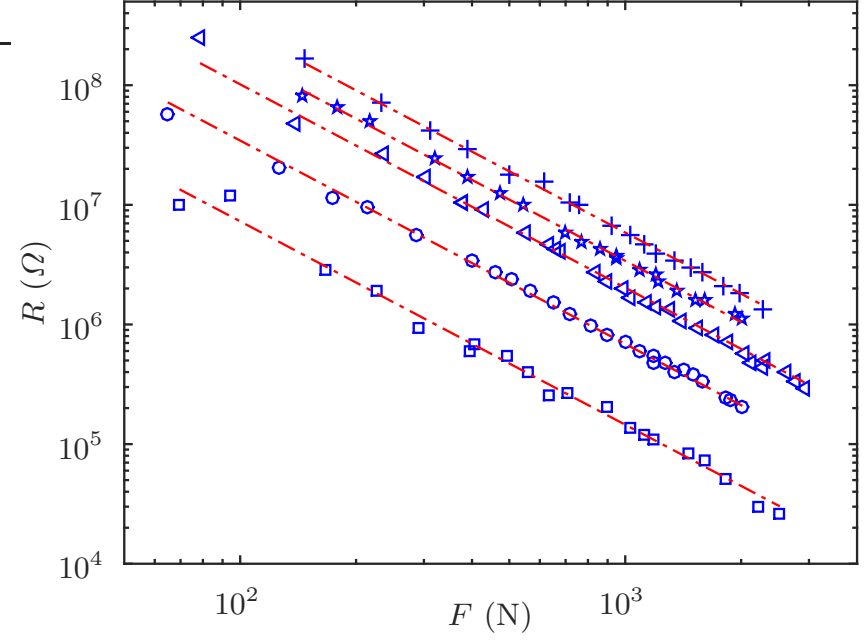

Fig. 5. Resistance of copper powder samples of different sizes versus the applied force $F$. Squares: $m=0.2 \mathrm{~g}, \Phi=7 \mathrm{~mm}$, $h=1 \mathrm{~mm}$, circles: $m=1 \mathrm{~g}, \Phi=7 \mathrm{~mm}, h=4.7 \mathrm{~mm}$, triangles: $m=2 \mathrm{~g}, \Phi=7 \mathrm{~mm}, h=9.4 \mathrm{~mm}$, stars: $m=3 \mathrm{~g}, \Phi=7 \mathrm{~mm}, h=$ $14.2 \mathrm{~mm}$, plusses: $m=9 \mathrm{~g}, \Phi=12 \mathrm{~mm}, h=14.5 \mathrm{~mm}$. Dashed lines are power laws with a slope $-\alpha_{p_{I}}=-1.7$.

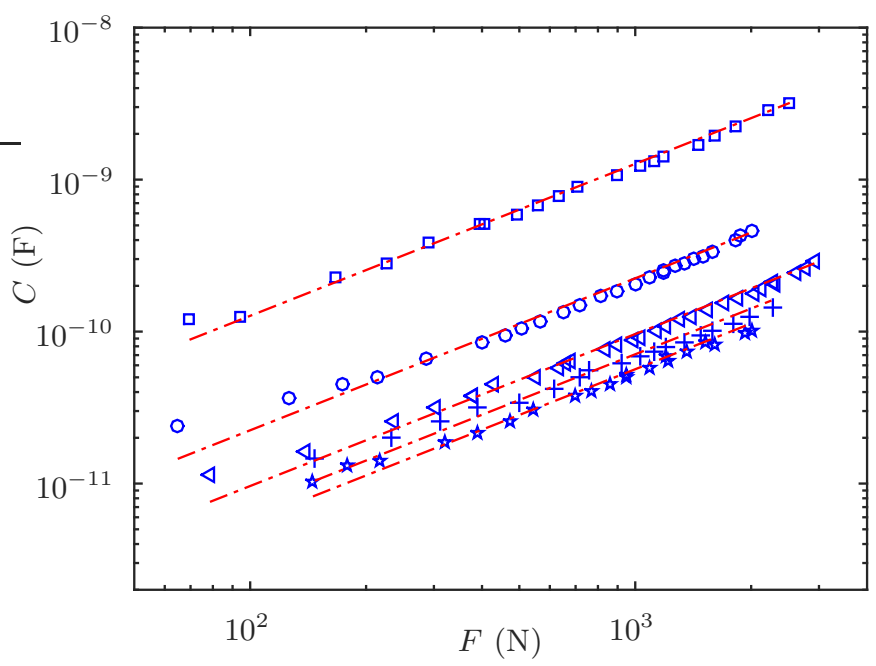

Fig. 6. Low frequency capacitance of copper powder samples of different sizes versus the applied force $F$. Symbols are as in Fig. 5. Dashed lines represent power laws with a slope equal to 1 .

Adopting the simple model of conduction between two grains presented in section 2 , the capacitance $(c)$ of one contact is thus proportional to the contact surface as

$$
c=\epsilon_{o x} A / \delta_{o x}
$$

where $\epsilon_{o x}$ is the electric permittivity of the oxide that covers the grains. Using equations (1) or (2), this very simple model of the electric contact between two grains predicts a power-law behaviour of the force-dependent capacitance:

$$
\begin{array}{ll}
c \propto f^{2 / 3} \quad(\mathrm{ER}), \\
c \propto f \quad(\mathrm{PR}) .
\end{array}
$$




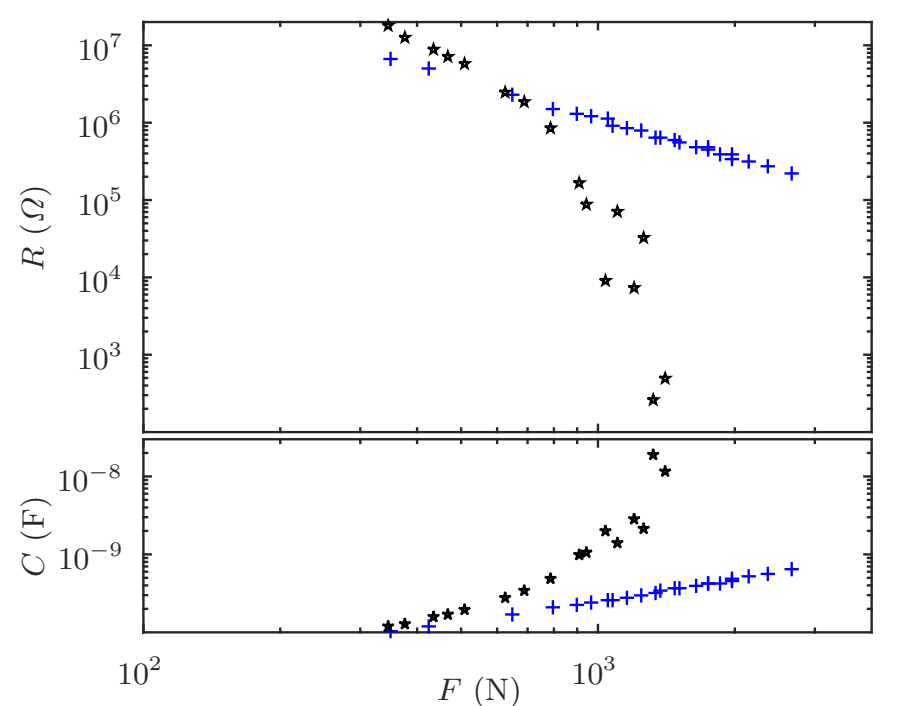

Fig. 7. Resistance and capacitance of samples from batch I (blue plusses) and from batch II (black stars) as a function of the force. Sample size: $m=2 \mathrm{~g}, \Phi=10 \mathrm{~mm}, h=4.6 \mathrm{~mm}$.

These above results lead to two quite different conclusions. First, a simple model of electrical conduction can explain well the experimentally observed capacitance for the powder although the number of grains is very large $(>100000)$ and the force network is much complex within the powder. As previously estimated in section 3, most of the contacts in the powder are described by Eq. (2). A large amount of the grains are thus deformed plastically by the mechanical force which imposes the change of the powder between two experimental runs. On the contrary, the force-dependent resistance remains unexplained and a simple model of conduction between two grains disagrees with the observed results for the powder. Assuming a contact area $(A)$ proportional to the force [Eq. (2)], Eq. (8) for two grains in contact becomes:

$$
r \propto A^{-\alpha_{p_{I}}}
$$

with $\alpha_{p_{I}}=1.7$ which is significantly greater than one.

\section{Results for powder from batch II}

The powder of batch II gives further interesting results. As seen in Fig. (7), the resistance of the powder from batch II does not depend as a power law of the force. Rather, the resistance falls rapidly when approaching a critical force. The capacitance also exhibits a complex dependence on the force: $C$ grows faster than $F$ [see also Fig. (7)], thus Eq. (9) is not valid for the powder from batch II. A clear interpretation is simply that for powders from batch II, as the force between the grains is very large, the grains are extremely plastic to such an extent that Eq. (2) is no longer valid. In this case, the pressure between the grains $p=F / A$ is no longer constant and even decreases when the force increases. Then the contact area $A$ increases more rapidly than the force, which reflects in

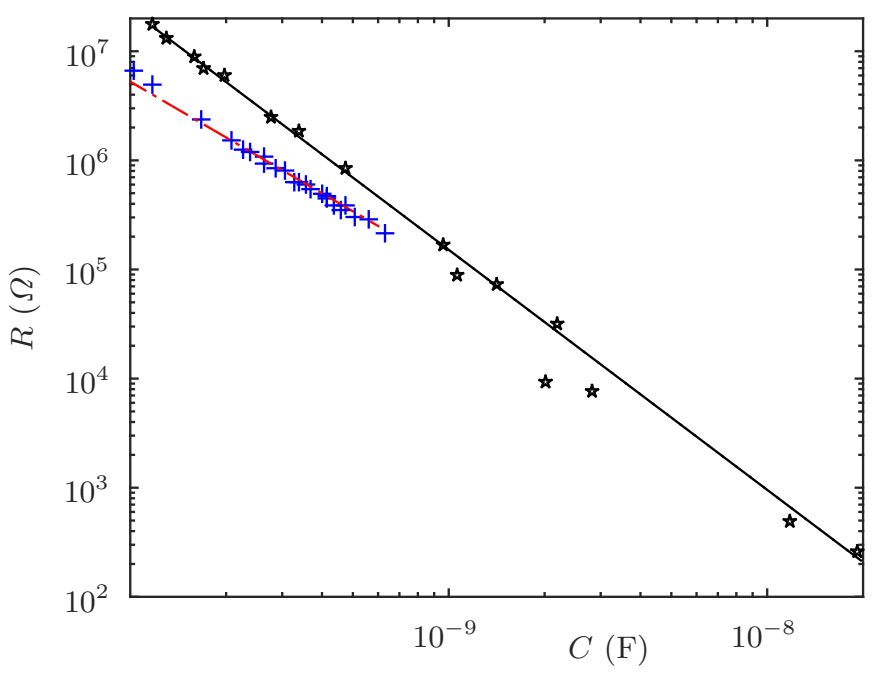

Fig. 8. Resistance of samples from batch I (blue plusses) and II (black stars) as a function of the capacitance. Red dashed line: $R \propto C^{-\alpha_{p_{I}}}$ with $\alpha_{p_{I}}=1.7$. Black solid line: $R \propto C^{-\alpha_{p_{I I}}}$ with $\alpha_{p_{I I}}=2.2$.

a spectacular increase in the capacitance when the force increases. At the same time, the capacitance stays proportional to the contact area as for the grains for the powder of batch I. Coming back to the force-dependent resistance and assuming a capacitance proportional to the contact area for the powder of batch I, Eq. (13) shows that the resistance must exhibit a power-law dependence on the capacitance with a slope $-\alpha_{p_{I}}$, and this result is well experimentally observed in figure 8 . Similarly, with the same assumption that is $c \propto A$, the resistance for powders of batch II is also a power law of the capacitance with a slope $-\alpha_{p_{I I}} \simeq-2.2$ which is close to $-\alpha_{p_{I}}$ (Fig. 8).

Despite a significant difference between the mechanical behaviours of the two powders explored in this paper, the electrical resistance when plotted against the capacitance is described by a power law for the two powders with an exponent $\alpha_{p_{I}}$ or $\alpha_{p_{I I}}$ both largely greater than one. Besides, these exponents cannot be easily explained by a simple model of conduction between two grains. Finally, it has to be emphasized the very good reproducibility of both the resistance and the capacitance of the powders. In other words, at fixed geometry (mass and diameter constants), the resistance and capacitance depend only on the force. We will see in Sect. 4.3 that this situation is different for the chain of beads.

\subsection{The powder seen as an effective medium}

The good reproducibility of the experimental measurements observed for both the resistance and the capacitance suggests that the powder can be considered as an effective medium, whose properties would depend on the applied pressure $P$ defined as

$$
P=\frac{4 F}{\pi \Phi^{2}}
$$




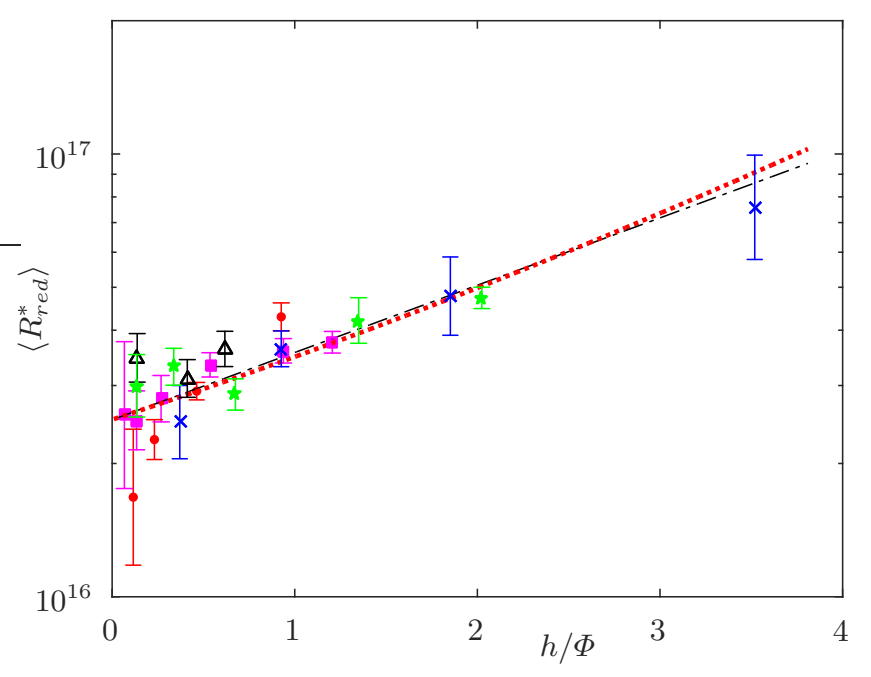

Fig. 9. Dependence of the averaged reduced resistivity on the aspect ratio $h / \Phi$ for powder samples from batch I. Blue crosses: $\Phi=5 \mathrm{~mm}$, green stars: $\Phi=7 \mathrm{~mm}$, red circles: $\Phi=10 \mathrm{~mm}$, purple squares: $\Phi=12 \mathrm{~mm}$, black triangles: $\Phi=15 \mathrm{~mm}$. Black dot-dashed line: $\left\langle R_{\text {red }}^{*}\right\rangle \propto \exp \left(0.35 \frac{h}{\Phi}\right)$ [Eq. (22)]. Red dotted line: Eq. (21) with $2 \alpha_{p_{I}} f_{w}=0.31$.

If so, this effective medium has an averaged resistivity $R^{*}(P)$, defined by the following equation:

$$
R^{*}(P) \equiv \frac{\pi \Phi^{2}}{4 h} R(P)
$$

$h$ and $\Phi$ are respectively the height and the diameter of the powder sample. Using the observed power-law dependence of the resistance versus $F$ [Eq. (8) is valid for powder samples from batch I], it results that the reduced resistivity

$$
R_{\text {red }}^{*} \equiv R^{*} P^{\alpha_{p_{I}}}
$$

should be independent, both of the sample geometry, and the applied force or pressure. At fixed geometry, the reduced resistivity has been averaged across all the measured force giving $\left\langle R_{\text {red }}^{*}\right\rangle$, and the result is plotted as a function of the aspect ratio $(h / \Phi)$ in a semi-logarithmic plot in Fig. 9. For a given aspect ratio, the reduced resistivity is found to be again well reproducible whatever the diameter or the mass of the powder sample. Secondly, the reduced resistivity has a clear exponential dependence on the aspect ratio. Next we show that this behaviour results from the existence of arches within the powder under compression, a well known phenomenon in granular matter called Janssen effect [7]. By contrast to a vertical cylinder filled with a liquid in which the lateral walls support none of the vertical load, for a cylinder filled with grains, the emergence of arches in the complex network of the forces between the grains redirects the weight towards the side walls. Hence, the pressure at the bottom within the granular material does not increase linearly with the height of the powder sample but rather saturates to a constant value.

At fixed geometry, the variation of the pressure in the powder $(p)$ as a function of the height can be calculated using the simplified model given by Janssen in his paper [7].
Consider a slice of the powder sample of diameter $\Phi$ and thickness $\mathrm{d} z$, located at a distance of $z$ from the bottom of the container. Imposing the mechanical equilibrium of this piece of granular material the following equation is obtained

$$
\frac{\pi \Phi^{2}}{4}[p(z)-p(z+\mathrm{d} z)-\mu g \mathrm{~d} z]=\pi \Phi p_{w} \mathrm{~d} z
$$

where $\mu$ is the density of the powder and $g$ is the gravity acceleration. The right hand-side of Eq. (17) represents the force of friction and $p_{w}$ the corresponding pressure applied by the lateral wall on the grains that are in contact with the container. This force balances both the difference of pressure across the slice of powder and the own weight of the slice. Next, assuming that the pressure applied by the walls is proportional to the vertical pressure $\left(p_{w}=\right.$ $\left.f_{w} p\right)$, Eq. (17) becomes:

$$
\frac{\mathrm{d} p}{\mathrm{~d} z}+\frac{4 f_{w} p}{\Phi}=-\mu g
$$

In fact, $f_{w}$ is the product of two different and phenomenological coefficients: $f_{w}=\mu_{s} \times K$. The coefficient of static friction $\left(\mu_{s}\right)$ is the ratio between the pressure applied by the walls and the horizontal pressure within the powder, whereas $K$ represents the ratio between the horizontal and the vertical pressures. Measuring these two coefficients is a great challenge and needs sophisticated experimental setups and procedures. To our knowledge, $\mu_{s}$ is not known for a copper surface sliding on walls made of PMMA. It also depends on the oxidation state and the surface roughness of the grains. As a rough order of magnitude, [21] found $0.3 \leq \mu_{s} \leq 0.4$ for stainless steel on a PMMA surface. The second coefficient $K$ is also not known and depends on the friction coefficient between two copper grains. But it also depends largely on the packing density $([22])$ and so on the history of the granular medium. [22] reported values of $K$ between 0.6 and 0.9 but they used glass beads. Anyway, for the Janssen's model, only the product of $K$ by $\mu_{s}$ plays a role in Eq. (18). Besides, hereafter, $f_{w}=K \mu_{s}$ is considered as a fitting parameter and can vary from 0 to 1 .

For the experiments considered here, the pressure measured at the bottom is always greater than $2.5 \mathrm{GPa}$ whereas $\mu g h<1 \mathrm{kPa}$. Hence the weight of the grains can be largely neglected as well as the right-hand side of Eq. (18). Besides, the coefficient $f_{w}$ is assumed to be both constant within the powder and independent of the force applied. Integrating Eq. (18) between $z=0$ (bottom) and $z$, the pressure profile is obtained

$$
p(z)=P \exp \left(-4 f_{w} \frac{z}{\Phi}\right)
$$

where $P=p(0)$ is the pressure measured at the bottom. Then, the resistance of a powder sample of height $h$ can be calculated as follow

$$
R=\frac{4}{\pi \Phi^{2}} \int_{0}^{h} \rho(p) \mathrm{d} z,
$$




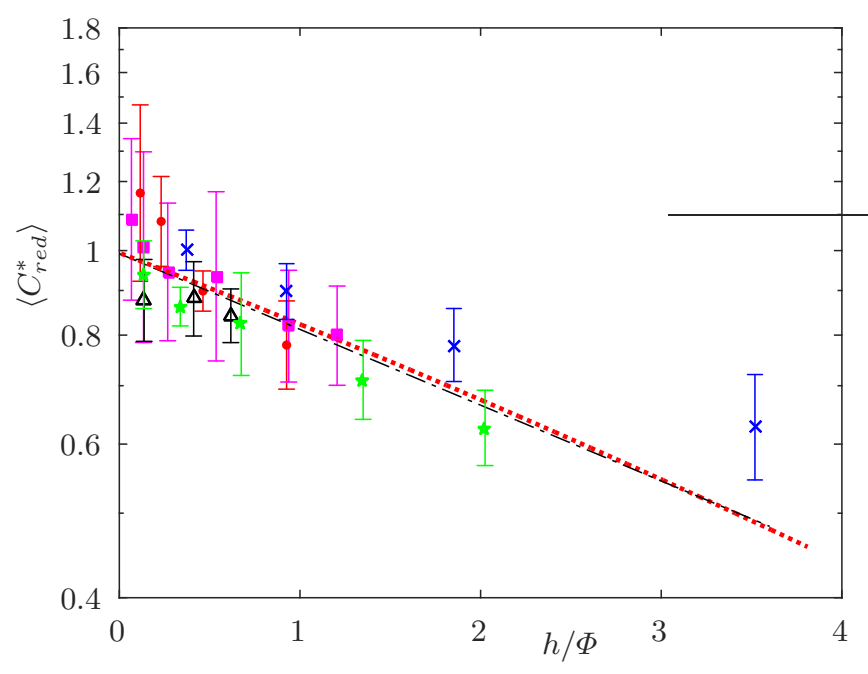

Fig. 10. Dependence of the averaged reduced capacitance on the aspect ratio $h / \Phi$ (powder from batch I). Symbols are as in Fig. 9. Black dot-dashed line: $\left\langle C_{\text {red }}^{*}\right\rangle \propto \exp \left(-0.2 \frac{h}{\Phi}\right)[$ Eq. (26)]. Red dotted line: Eq. (25) with $2 f_{w}=0.18$.

with $\rho$ the resistivity of the powder depending on the coordinate $z$ or else on the local pressure. According to Eq. (8), the reduced resistivity is constant: $\rho_{\text {red }} \equiv \rho p^{\alpha_{p_{I}}}=$ $\rho(0) P^{\alpha_{p_{I}}}$. Integrating Eq. (20), we obtain

$$
\begin{aligned}
R_{\text {red }}^{*} & =\rho_{\text {red }} \frac{\sinh \left(2 \alpha_{p_{I}} f_{w} \frac{h}{\Phi}\right)}{2 \alpha_{p_{I}} f_{w} \frac{h}{\Phi}} \exp \left(2 \alpha_{p_{I}} f_{w} \frac{h}{\Phi}\right), \\
& \approx \rho_{\text {red }} \exp \left(2 \alpha_{p_{I}} f_{w} \frac{h}{\Phi}\right) \text { for } f_{w} \frac{h}{\Phi} \ll 1 .
\end{aligned}
$$

Hence, this model of arches within the powder shows that the electrical resistivity measured experimentally is not far away from an exponential function of the aspect ratio. Equations (21) and (22) are plotted in Fig. 9 with respectively $f_{w}=0.09$ and $f_{w}=0.10$ as fitting parameters.

In the same way, the capacitance can be calculated as

$$
C^{-1}=\frac{4}{\pi \Phi^{2}} \int_{0}^{h} \frac{\mathrm{d} z}{\epsilon(p)},
$$

where $\epsilon$ is the electric permittivity of the powder. As the capacitance is observed to be proportional to the force or the pressure applied to the powder [Eq. (9) is valid for powder samples from batch $\mathrm{I}]$, the reduced electric permittivity is constant: $\epsilon_{\text {red }} \equiv \epsilon p^{-1}=\epsilon(0) P^{-1}$. Integrating Eq. (23), we obtain

$$
\begin{aligned}
C_{r e d}^{*} & =\frac{4 h}{\pi \Phi^{2}} \frac{C}{P} \\
& =\epsilon_{r e d} \frac{2 f_{w} \frac{h}{\Phi}}{\sinh \left(2 f_{w} \frac{h}{\Phi}\right)} \exp \left(-2 f_{w} \frac{h}{\Phi}\right), \\
& \approx \epsilon_{r e d} \exp \left(-2 f_{w} \frac{h}{\Phi}\right) \text { for } f_{w} \frac{h}{\Phi} \ll 1 .
\end{aligned}
$$

Equations (25) and (26) are plotted in Fig. 10 with respectively $f_{w}=0.09$ and $f_{w}=0.10$. A very good agreement is observed with the experimental measurements showing

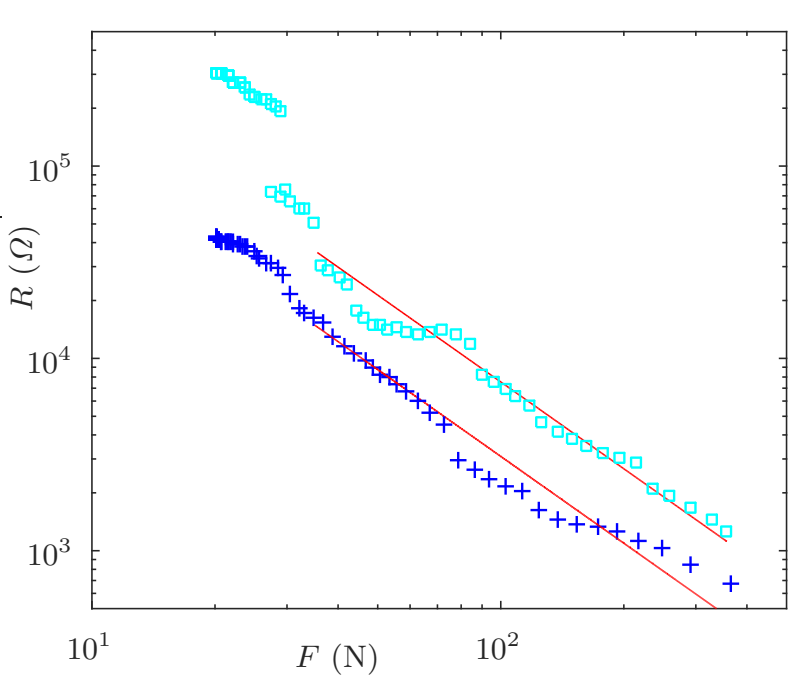

Fig. 11. Log-log plot of the electrical resistance as a function of the applied force for two different chains of 12 beads. The applied force is gradually increased while the electrical resistance is measured. Each run lasted around 20 minutes. Solid lines represent power laws with a slope of -1.5.

that the Janssen's model explains well the variation of the capacitance versus the aspect ratio.

Thus, to conclude this paragraph, the powder behaves like an effective medium and the very good reproducibility of the experimental measurements comes undoubtedly from the large number of grains within the powder. In section 6 , theoretical considerations are given to calculate the size (or the number of grains) needed in order to consider the powder as an effective medium.

\subsection{Results for the chain of beads}

As emphasized in Sect. 1, the strong dependence of the effective resistivity on the pressure observed in the case of metallic powders may be due to either a progressive rearrangement of the complex and three-dimensional map of the conducting channels in the powder, or to the average evolution of the contact resistance between the grains. The one-dimensional experimental set-up allows to study the contact between two grains in the case of only one conducting channel represented by the chain of beads. The experimental results presented below suggests that the power-law behaviour of the powder resistance with a large exponent $\left(\alpha_{p_{I}}, \alpha_{p_{I I}}>1\right)$ is a consequence of a power-law behaviour of the contact resistance between two grains with also an exponent greater than one.

In Sect. 3.2, we have explained that, thanks to the onedimensional set-up well instrumented with a step motor controlled by a computer and both the force sensor and the resistance meter also connected to the same computer, there are in fact two protocols to measure the electrical resistance of the beads as a function of the applied force. The first one is to increase the force from 0 to a maximum value of around $400 \mathrm{~N}$, and at the same time both this force and the electrical resistance of the chain of beads 


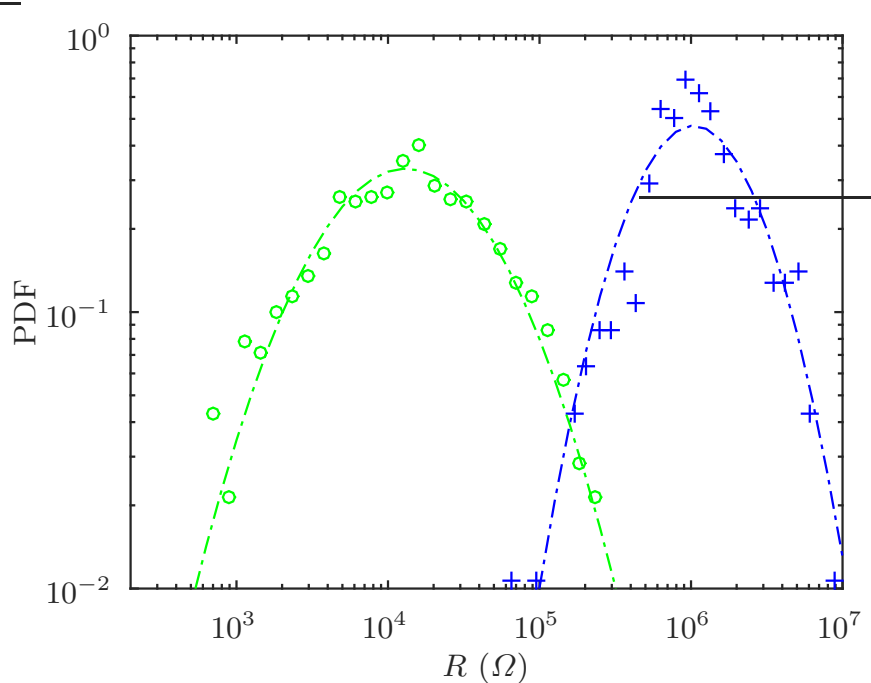

Fig. 12. Probability density function of the logarithm of the resistance for two different forces: $F=20 \mathrm{~N}$ (blue plusses) and $F=400 \mathrm{~N}$ (green circles). The chain is constituted by 21 beads which corresponds to 20 bead-bead contacts. Dashed lines represent log-normal laws.

are measured and recorded. Figure 11 shows two measurements of the resistance using this protocol. For each run, one can see that when the force increases, the electrical resistance does not decay regularly. Indeed, sometimes the resistance drops suddenly whereas the force increases very little. In this case, we have never observed any external perturbation or large variations of the mechanical properties of the chain of beads. For example, we have recorded the chain displacement $\delta_{d}$ at the same time as the electrical resistance. $\delta_{d}$ is found to be a power law of the force with an exponent $2 / 3$ without any sudden and abrupt jumps of $\delta_{d}$. The mechanical behaviour of the beads is very well described by the Hertz's law (also reported in [19]) whereas the electrical behaviour is more complex and certainly cannot only be explained by an increase of the contact surface when the force between two beads increases. The oxide film which covers each bead plays surely a crucial role for the electrical resistivity of the contact between two beads whereas it has no effect on the mechanical behaviour of the chain of beads.

All the same, forgetting for the moment the sudden drops of the resistance, $R$ as a function of $F$ can be fitted by a power law with a slope between -1.6 and -1.3 . In Fig. 11, a $F^{-1.5}$ scaling is plotted as a visual indication. Contrary to the results for the powder presented previously, it is clearly not possible with this method to measure with a good precision the slope since the power-law behaviour is just a rough approximation of the dependence of $R$ on the force. And also at a given force, the resistance varies widely between two chains of 12 beads whereas the resistance of the powder depends only on the weight of the powder (or on the number of grains) and the force.

Then, the second kind of experiments we have done was to fix the force and to measure several times the electrical resistance of the chain of beads. Obviously, it would

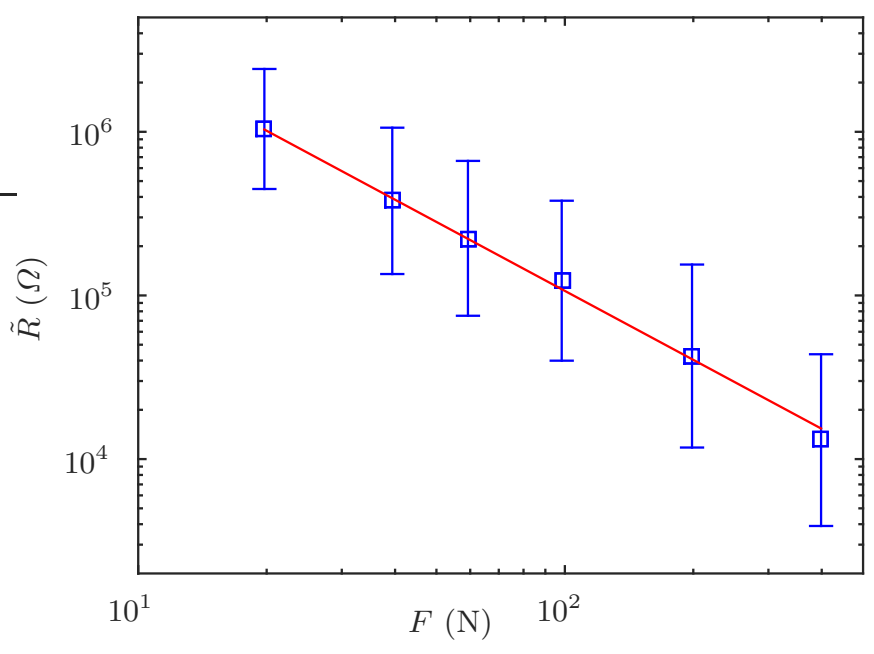

Fig. 13. Log-log plot of the resistance $\tilde{R}=\exp (\langle\ln R\rangle)$ as a function of the applied force for a chain of 21 beads. For each force, $\tilde{R}$ is obtained by averaging around 600 measurements of the resistance. Each errorbar represents the corresponding standard deviation $\sigma$ calculated over the same measurements. Solid line represents a power-law fit with a slope of -1.4 .

have been costly and time-consuming to change every time the beads after each measurement. But, we have noticed that rolling the beads along the chain axis is sufficient in order to test a new and fresh contact between the beads. In [19], the authors showed that this technique ensures reproducible measurements. So, to investigate a possible power-law behaviour of the electrical resistance as a function of the force, we have spent many hours to measure many times the resistance of one chain of beads at fixed force. Between two measurements, the force has been decreased, the piston has been driven back and we have systematically rolled the beads. Then, the piston has again been pushed on the chain of beads and the force has been increased to the prescribed value. For each value of the investigated force, we have measured around 600 times the electrical resistance of the chain of beads. Figure 12 shows the probability density function of the logarithm of the resistance for two different forces: $F=20 \mathrm{~N}$ (plusses) and $F=400 \mathrm{~N}$ (circles). At fixed force, the resistance varies over more than two orders of magnitude and the probability density function of the resistance can well be fitted by a $\log$-normal distribution with parameters $\langle\ln R\rangle$ and $\sigma^{2}=\left\langle(\ln R-\langle\ln R\rangle)^{2}\right\rangle$ which are respectively the mean and the standard deviation of the logarithm of the resistance. Naturally, $\langle\ln R\rangle$ and $\sigma$ depend on the force applied. In Fig. 13, the characteristic resistance $\tilde{R}=\exp (\langle\ln R\rangle)$ is plotted against the force. Similarly to the experiments with the powder but using $\tilde{R}$, the resistance is very well fitted by a power law

$$
\tilde{R} \propto F^{-\alpha_{b}}
$$

In the case of a chain of 21 beads that is 20 contacts between two beads, the exponent of this power law is equal to $\alpha_{b}=1.4$ whereas for the powders, the obtained exponent is equal to $\alpha_{p_{I}}=1.7$ or $\alpha_{p_{I I}}=2.2$. Anyway, the two 
experimental systems are very different granular media (1D vs. 3D, 20 vs. more than 100000 particles, in stainless steel vs. copper ...) but in both cases, they exhibit a similar behaviour that is a strong dependence of the resistance with the force: $\alpha_{p_{I}}, \alpha_{p_{I I}}, \alpha_{b}>1$. We can finally note that, according to the Hertz's law [Eq. (1)], the resistance of the chain of beads is a power law of the contact area: $\tilde{R} \propto A^{-3 \alpha_{b} / 2}$, with an exponent $3 \alpha_{b} / 2=2.1$, whereas for the powder this exponent is equal to $\alpha_{p_{I}}=1.7$ since most of the grains from batch $\mathrm{I}$ are in the plastic regime.

\section{The proposed model}

The main result obtained in the last section is that the electrical resistance decreases much faster than the increasing contact area when the mechanical force is increased for both the metallic powders and the chain of beads. Hence, to model this striking effect observed both in $1 \mathrm{D}$ and $3 \mathrm{D}$ granular materials, we cannot refer to some percolation theories or to the complex network of the forces between the grains in the powder since in the $1 \mathrm{D}$ experiment, there is only one conduction channel and the beads are compressed by the same force. On the contrary, the capacitance increases like the contact area for the powder and the behaviour of the capacitance is well explained by a very simple model that is $c$ is proportional to the contact area and inversely proportional to the thickness of the oxide that covers the beads [Eq. (10)].

To interpret this observed difference of behaviour between the resistance and the capacitance, a more detailed description of the contact between two grains is needed. To focus our attention on the contact, the sketch of two grains in Fig. 1 is zoomed in Fig. 14. At this scale, the oxide and maybe some impurities that cover the grains are not homogeneous which also leads to a non uniform resistivity of this oxide film. Hence, when a voltage is applied, the electric current $(I)$ does not flow uniformly through the oxide. Rather, it follows a given number of conducting micro-channels for which the resistivity is low due to a better conductivity of the oxide in these places. Besides, it is reasonable to assume that, on the one hand the number of micro-channels $(k)$ increases proportionally with the contact area $(k \propto A)$, on the other hand their resistivity follows a given distribution characterizing the properties of the oxide or impurities present at the surface of the grains. As the micro-channels are connected in parallel and not in series, it is better to define the probability density function $(Q)$ of the conductances $(\gamma)$ of the micro-channels. Then, if $Q$ is such that $\gamma$ admits an average value, the resistance can be written as

$$
\begin{aligned}
\frac{1}{R} & \equiv \Gamma=\sum_{i=1}^{k} \gamma_{i} \\
& \approx k \int_{0}^{\infty} Q(\gamma) \gamma \mathrm{d} \gamma=k\langle\gamma\rangle
\end{aligned}
$$

Hence, the electrical resistance is found to be again inversely proportional to the contact area as for a uniform

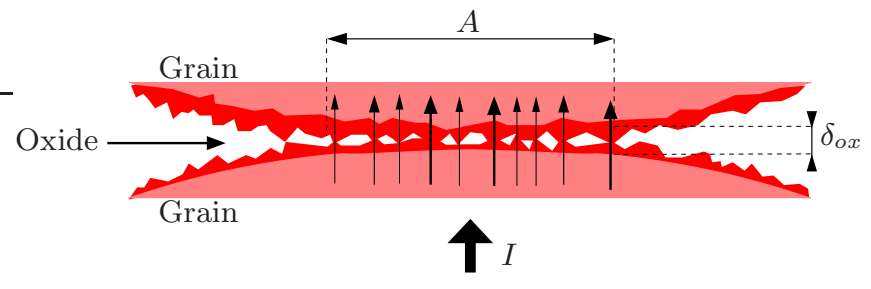

Fig. 14. Schema of the contact between two grains. The grains are covered of non-homogeneous oxide and impurities. The current $I$ is then divided into multiple "micro-currents" that follow conducting micro-channels.

oxide layer, and thus $R \propto F^{-2 / 3}$ in the elastic regime and $R \propto F^{-1}$ in the plastic regime, the exponents are again far away from the ones obtained experimentally.

Another possibility not yet explored is to assume that the oxide layer is strongly non-homogeneous. This may come from the large variability of the characteristics of the micro-channels (nature of the oxide, length, width...). In this case, the distribution of the conductances can be very broad leading to a non finite mean of the distribution: $\langle\gamma\rangle=\infty$. The Pareto distribution is an example of this kind of distribution:

$$
Q(\gamma)=\mu \frac{\gamma_{o}^{\mu}}{\gamma^{1+\mu}},
$$

the parameter $\mu$ controlling the tail of $Q$. Large values of $\mu$ give narrow distributions of $\gamma$ whereas values of $\mu$ close to zero give broader distributions. The degree of disorder and the variability increase when $\mu$ decreases. Hereafter, $0<\mu<1$ so that $\langle\gamma\rangle$ does not exist. The standard central limit theorem does not apply, but its generalization states that the sum of $k$ independent conductances tends to a $k$-independent Lévy distribution $\Psi$ [23,24]:

$$
\Gamma=k^{1 / \mu} \Psi
$$

with $1 / \mu>1$. In the plastic regime, when the force increases, both the contact area and the number of microchannels increase proportionally to the force $(k \propto A \propto f)$, but the total conductance of the contact increases faster than the force: $\Gamma \propto f^{1 / \mu}$.

To illustrate this model in the case of the chain of beads, we can simulate the electrical resistance of two beads in contact as a function of the force using the Pareto distribution [Eq. (30)]. The coefficient $\mu_{b}$ has to be chosen such that $1 / \mu_{b}=3 \alpha_{b} / 2$ yielding to the value of 0.47 for $\mu_{b}$ but we prefer to fix $\mu_{b}=0.44$ in order to fit better the experimental results. Indeed, we will show that the slope of the power-law behaviour of the resistance with the force [Eq. (27)] depends slightly on the number of beads (see Fig. 16). In fact, it decreases a bit when the number of beads increases yielding to $\alpha_{b}=1.5$ for two beads in contact and $\alpha_{b}=1.4$ for 21 beads in contact. As for the number of micro-channels, $k$ is not experimentally measured. But, in practice, $k$ can be chosen arbitrary, at least while respecting $k \propto A \propto F^{2 / 3}$. Here, $k=6,20$ and 60 yielding to $F=k^{3 / 2} \simeq 15,90$ and $465 \mathrm{~N}$, respectively. Then for 


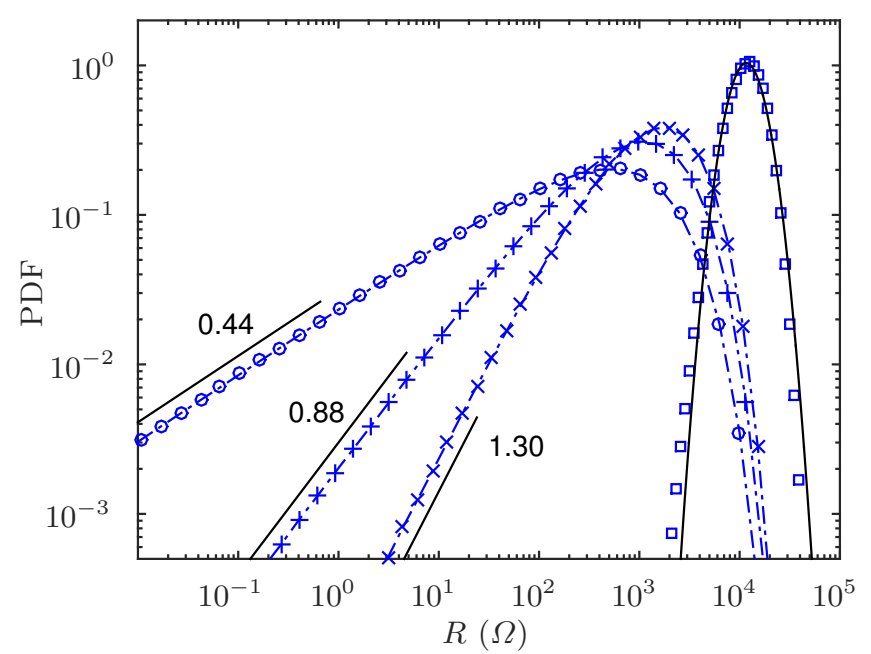

Fig. 15. Results from the model. Probability density functions of the logarithm of the resistance for two beads in contact $\psi_{1}$ (circles), three beads or two bead/bead contacts $\psi_{2}$ (plusses), four beads $\psi_{3}$ (crosses) and 21 beads (squares). Straight lines are power-law fits of the tails of the PDFs for 2, 3 and 4 beads with slopes $0.44,0.88$ and 1.31 respectively. PDF for the chain of 21 beads is fitted by a gaussian law (solid line) that also corresponds to a log-normal law for the resistance. The number of micro-channels is here fixed to $k=60$ that corresponds to a fixed force of $465 \mathrm{~N}$.

each force, the values of the $k$ conductances are given randomly by the Pareto distribution. The constant $\gamma_{0}$ is the only one parameter needed to be adjusted to fit the experimental data. The obtained conductance follows a Lévy distribution [Eq. (31)] that is shown in Fig. 15 (circles). Actually, the probability density function of the logarithm of the obtained resistance is plotted in this figure and the tail of this distribution follows a power law with a slope equal to $\mu_{b}=0.44: \psi_{1}(\ln R) \propto R^{\mu_{b}}$ when $R<1 \Omega$.

Then, for each force, the electrical resistance of three beads in contact can be directly calculated as the sum of the resistances of two bead/bead contacts. The obtained distribution of the logarithm of the resistance $\left(\psi_{2}\right)$ is plotted in Fig. 15 (plusses). This distribution is narrower than $\psi_{1}$ with also a steeper tail: $\psi_{2} \propto R^{0.88}$. For a chain of 21 beads in contact, the distribution is nearly symmetrical around its maximum and is close to a normal law or a log-normal law for the resistance, as observed experimentally in Fig. 12. The characteristic resistances $\tilde{R}=\exp (\langle\ln R\rangle)$ obtained experimentally and numerically are plotted against the force in Fig. 16 for two beads in contact (circles) and also for a chain of 21 beads (squares). There is a quite good agreement between the experimental values and the model for the two systems. In particular, the model describes very well the power-law behaviour of the resistance with the force and also the non-linear evolution of the resistance with the number of beads at a given force (This is discussed in further detail in next section). On the contrary, the model under-estimates the standard deviation of $\ln R$ for the chain of 21 beads. Finally, the model shows that the slope of the power law $\tilde{R} \propto F^{-\alpha_{b}}$

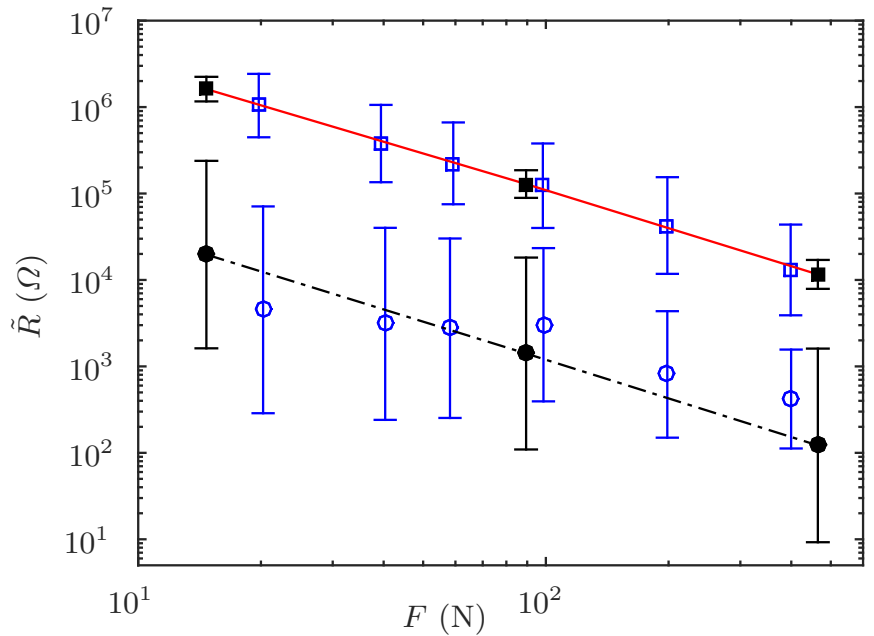

Fig. 16. $\log -\log$ plot of the resistance $\tilde{R}=\exp (\langle\ln R\rangle)$ as a function of the applied force for two beads in contact (circles) and for a chain of 21 beads (squares). Open symbols represent experiments and plain symbols are given by the model. Each errorbar represents the corresponding standard deviation $\sigma=\sqrt{\left\langle(\ln R-\langle\ln R\rangle)^{2}\right\rangle}$. Solid line: slope -1.5. Dot-dashed line: slope -1.4 .

depends a bit on the number of beads: $\alpha_{b}=1.5$ for two beads and $\alpha_{b}=1.4$ for 21 beads.

\section{Random lattice and effective medium}

This section is dedicated to the evolution of the electrical resistance of both the chain of beads and the powder with the number of beads or grains. As observed in previous sections, the slope $\alpha_{b}$ for the chain of beads depends on the number of beads whereas, for the powder, the slope $\alpha_{p}$ does not depend on the size of the sample. Furthermore, even for a chain with 21 beads, the resistance at a fixed force is given by a log-normal law with a large standard deviation whereas the resistance of a powder sample is very reproducible.

\subsection{The chain of beads ( 1 dimension)}

The previous model well explains the unusual behaviour of the electrical resistance with the force for the two metallic granular media experimentally investigated in this paper. But the model also shows that the contact resistance between two grains is controlled by a few random conductance channels. For the chain of beads, this implies that, at a given force, the resistance varies a lot when the beads are changed or moved in order to "refresh" the contacts. Besides, the model also implies that the resistance does not depend linearly with the number of beads $(N)$ or the number of contacts $N_{c}=N-1$ (see Fig. 17). All these features are observed experimentally for the chain of beads, the standard deviation $\sigma\left(N_{c}\right)=\sqrt{\left\langle(\ln R-\langle\ln R\rangle)^{2}\right\rangle}$ measured experimentally is even greater than the one obtained 


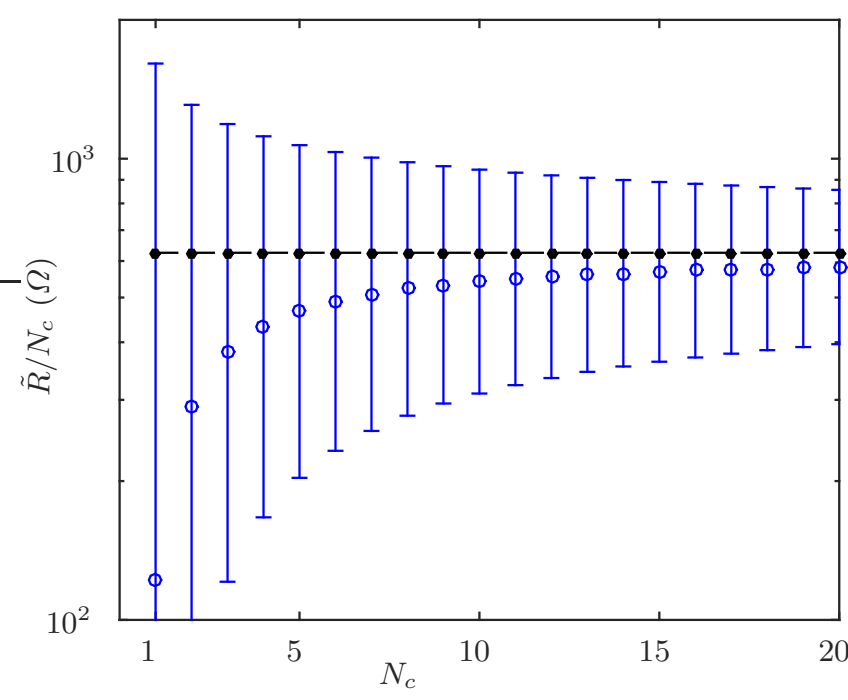

Fig. 17. Model for the chain of beads: plot of the resistance $\tilde{R}$ divided by the number of contacts as a function of $N_{c}$. The force is fixed at $400 \mathrm{~N}$. Each errorbar represents the corresponding standard deviation $\sigma\left(N_{c}\right)=\sqrt{\left\langle(\ln R-\langle\ln R\rangle)^{2}\right\rangle}$. Horizontal line: asymptotic value of $\tilde{R} / N_{c}$ when $N_{c} \rightarrow \infty$. Black points: $\langle R\rangle / N_{c}$ versus $N_{c}$.

by the model when the number of contacts is 20 , showing that the distribution of the resistance stays large even when the resistance is averaged over 20 contacts. Obviously, $\sigma\left(N_{c}\right)$ decreases when $N_{c}$ increases but it decreases slowly while the resistance per contact converges slowly to its asymptotic value $\left(\tilde{R} / N_{c}\right)_{N_{c} \rightarrow \infty}$.

For the chain of beads, the total resistance of $N_{c}$ contacts is always the sum of the $N_{c}$ resistances of contact so that, when the average resistance $\langle R\rangle$ is finite, $\langle R\rangle / N_{c}$ is independent of the number of contacts and hence :

$$
\frac{\langle R\rangle}{N_{c}}=\left(\frac{\langle R\rangle}{N_{c}}\right)_{N_{c} \rightarrow \infty} \equiv R_{e f f} .
$$

$R_{e f f}$ is called the effective resistance of the chain of beads and depends only on the electrical properties of the contact between two beads. Then, for the stainless steel beads experimentally investigated in this paper, a chain of those beads can be considered as an effective medium when $\tilde{R} / N_{c}=R_{e f f}$. Data from Fig. 17 can be plotted differently by focusing on the effect of the standard deviation $\sigma$ on the difference between the logarithm of the asymptotic resistance and the mean of the logarithm of the resistance:

$$
\delta \equiv \ln R_{e f f}-\left\langle\ln \left(R / N_{c}\right)\right\rangle
$$

$\delta$ increases with $\sigma$ (see Fig. 18) but it also depends on the form of the distribution of the individual resistances. Indeed, for a log-normal law for the distribution of the contact resistances, $\delta$ increases as $\sigma^{2} / 2$ (solid line in Fig. 18). For a skewed distribution and neglecting the terms of order higher than $\sigma^{3}$, using Eqs. (32) and (33) one can obtain:

$$
\delta=\sigma^{2} / 2+S \sigma^{3} / 6
$$

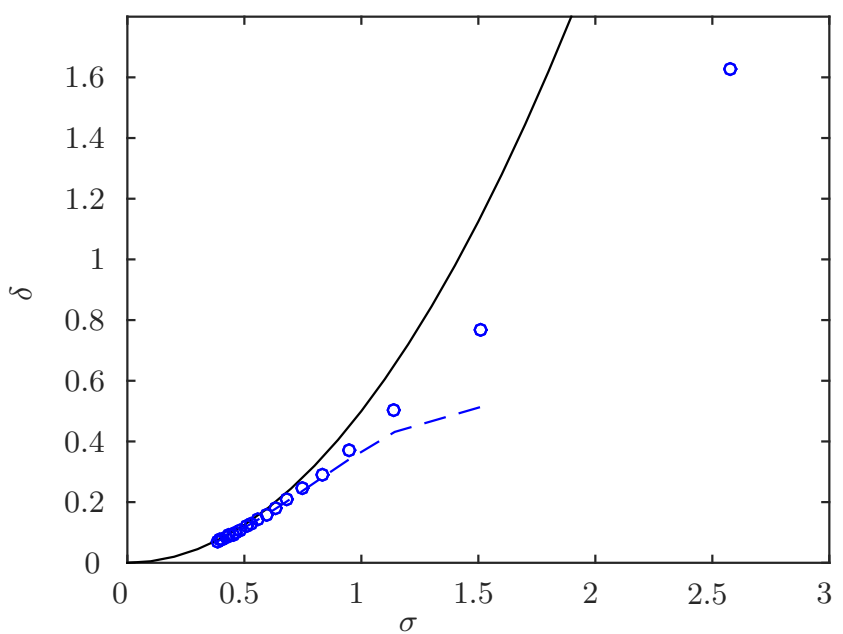

Fig. 18. Model for the chain of beads: difference between the logarithm of the asymptotic resistance and the mean of the logarithm of the resistances (circles). Solid line: $\delta$ obtained for a log-normal distribution for the contact resistances. Dashed line: Eq. (34).

$S$ being the skewness of the distribution. Obviously, this development of $\delta$ as a power series of $\sigma$ is valid when $\sigma$ is lower than 1. Indeed, for $\sigma>1$, large differences are observed between $\delta$ calculated numerically and Eq. (34).

\subsection{Two-dimensional systems}

Unlike the chain of beads for which the mean or "effective" resistance for a long chain is obtained easily thanks to Eq. (32), the situation is much more complex for granular packings at two or more dimensions. Indeed, in general, the effective resistance of $2 \mathrm{D}$ or $3 \mathrm{D}$ packings of metallic beads is not equal to the mean of the distribution of the resistances of one contact bead/bead. The effective medium theory (EMT) is a standard approximation for conduction in mixtures. For resistor networks and resistances distributed according to a distribution function $\phi(r)$, Kirkpatrick $[25,26]$ showed that the effective resistance $\left(r_{e f f}\right)$ is the solution of the equation:

$$
\int_{-\infty}^{\infty} \frac{r-r_{e f f}}{r_{e f f}+(z / 2-1) r} \phi(r) \mathrm{d} r=0,
$$

where $z$ is the number of bonds meeting at each node of the network. Obviously, for the chain of beads wherein $z=2$, Eq. (35) becomes $r_{\text {eff }}=\langle r\rangle$ : the effective resistance of one dimensional network is given by the mean of the distribution of the resistances without any approximation. But, for $2 \mathrm{D}$ or $3 \mathrm{D}$ systems, calculating the effective resistance is much more complicated in general. Indeed, $r_{\text {eff }}$ depends not only on the mean resistance but also on the higher moments of the distribution $\phi(r)$.

In the case of two-dimensional systems and for $z=4$, Eq. (35) can be rewritten as

$$
\int_{-\infty}^{\infty} \tanh \left(\frac{\sigma u-\delta}{2}\right) \Phi(u) \mathrm{d} u=0,
$$


with $u=(\ln r-\langle\ln r\rangle) / \sigma, \sigma^{2}=\left\langle(\ln r-\langle\ln r\rangle)^{2}\right\rangle$, and

$$
\delta \equiv \ln r_{e f f}-\langle\ln r\rangle .
$$

\subsubsection{For not very wide distributions $(\sigma<1)$}

For $\sigma<1$, a first order approximation of Eq. (36) gives $\delta=0$ or else

$$
r_{e f f} \approx \exp (\langle\ln r\rangle)
$$

In order to evaluate the limits of this equation, an iterative process can be used to calculate numerically the effective resistance of a large 2D lattice of resistors. This process is inspired by the renormalization group method and is described in appendix A.1. The results obtained numerically are compared with the Eqs. (36) and (38) in Fig. 19. For resistances at small scale following a log-normal law (or normal law for $\ln r$ ), the iterative process finds an effective resistance equal to $\exp (\langle\ln r\rangle)$ for a large piece of the material, whatever the width of the distribution, in agreement with Eq. (38) and, thus, also in agreement with Eq. (36). Indeed, for any symmetrical distribution $\Phi(u)$, Eq. (36) gives Eq. (38) without approximation. On the contrary, for a skewed distribution $\Phi, r_{e f f}$ is not strictly equal to $\exp (\langle\ln r\rangle)$. To show this, for each value of $\sigma$, we have generated a skewed distribution from a gaussian random variable $(g)$ of standard deviation $\sigma$. First, from $g$, we considered $\lg =\ln [\cosh (g)]-\langle\ln [\cosh (g)]\rangle$ and $w_{\sigma, S}=\alpha_{\sigma, S} g+\beta_{\sigma, S} \lg$. The two parameters $\alpha_{\sigma, S}$ and $\beta_{\sigma, S}$ are chosen to have, respectively, $\sigma$ and $S \sigma^{3}$ the standard deviation and the skewness of the skewed variable $w_{\sigma, S}\left(\left\langle w_{\sigma, S}^{2}\right\rangle=\sigma^{2}\right.$ and $\left.\left\langle w_{\sigma, S}^{3}\right\rangle / \sigma^{3}=S\right)$. Figure 19 indicates that for skewed distributions, $\delta$ deviates from zero when the standard deviation increases showing that the approximation $r_{\text {eff }} \approx \exp (\langle\ln r\rangle)$ becomes not valid for very broad and skewed distributions.

Using a third order approximation of Eq. (36), the effective medium theory leads to

$$
\frac{\delta}{\sigma}=\frac{4}{\hat{\sigma}} \cos \left\{\frac{1}{3}\left[\arccos \left(\frac{S \hat{\sigma}^{3}}{16}\right)-2 \pi\right]\right\},
$$

with $\frac{2}{\hat{\sigma}}=\sqrt{\frac{4}{\sigma^{2}}-1}$. Equation (39) is also plotted in Fig. 19 (dot-dashed lines) showing that this level of approximation of Eq. (36) remains valid as soon as $\sigma$ is lower than 0.3 for a skewed distribution with $|S|=1$. When $\sigma$ increases, we cannot neglect the moments of $\Phi(u)$ higher than the third moment and thus greater differences are seen between the Kirpatrick's equation and the approximation (39).

Next paragraph deals with broader distributions $(\sigma>1)$ and the link between effective medium theory and percolation theory.

\subsubsection{From very wide distributions to percolation theory}

Figure 20 shows that for the two skewed distributions of resistances (squares: $S=1$, circles: $S=-1$ ), $\delta / \sigma$ converges to an asymptotic value when $\sigma$ increases much

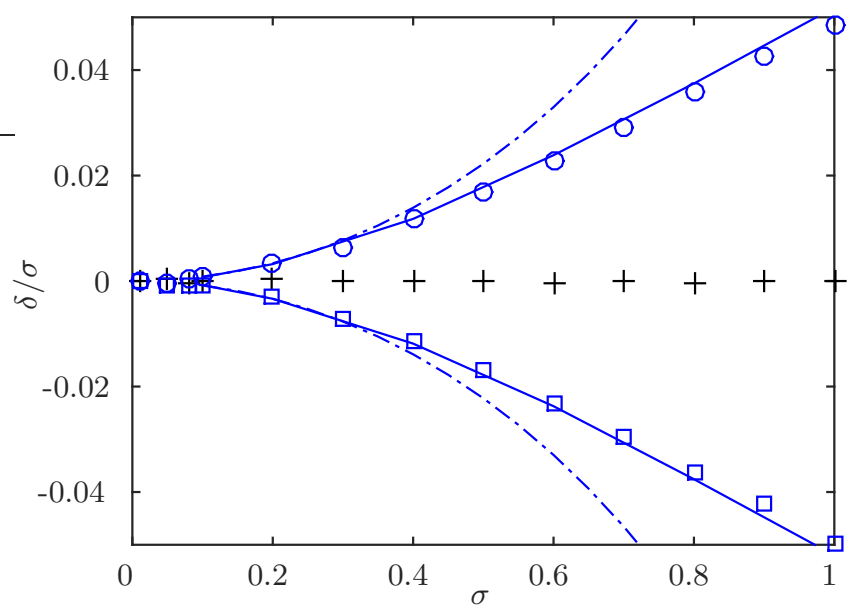

Fig. 19. Results from the iterative process and comparison with Eqs. (36) and (38). $\delta=\ln r_{e f f}-\langle\ln r\rangle$ is the difference between the logarithm of the effective resistance observed at large scale and the mean of the logarithm of the resistances at small scale. Plusses: $\delta / \sigma$ obtained for a normal distribution $\Phi(u)$ or a log-normal distribution for $\phi(r)$. Squares: results for a skewed distribution with $S=1$ whatever $\sigma$. Circles: results for a skewed distribution with $S=-1$. Solid lines: Eq. (36). Dot-dashed lines: Eq. (39).

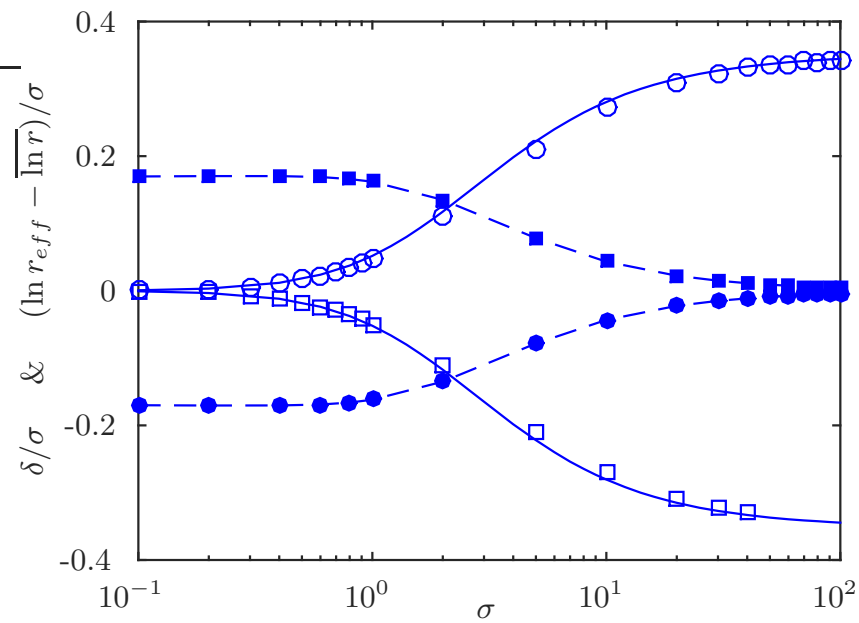

Fig. 20. $\delta / \sigma$ versus $\sigma$ (same symbols as Fig. 19). Solid lines: Eq. (36). Also plotted: median $(\overline{\ln r})$ of the distribution $\tilde{\Phi}(\ln r)$. Plain squares: $\left(\ln r_{e f f}-\overline{\ln r}\right) / \sigma$ for a skewed distribution with $S=1$ whatever $\sigma$. Plain circles: $\left(\ln r_{e f f}-\overline{\ln r}\right) / \sigma$ for a skewed distribution with $S=-1$.

larger than one. Indeed, Eq. (36) can be rewritten as

$$
\int_{-\infty}^{\infty} \tanh \left(\frac{\ln r-\ln r_{e f f}}{2}\right) \tilde{\Phi}(\ln r) \mathrm{d} \ln r=0 .
$$

When $\sigma$ tends to infinity, (40) can be approximated by

$$
\int_{-\infty}^{\ln r_{e f f}} \tilde{\Phi}(\ln r) \mathrm{d} \ln r=\int_{\ln r_{e f f}}^{\infty} \tilde{\Phi}(\ln r) \mathrm{d} \ln r
$$


showing that $\ln r_{\text {eff }}$ becomes the median of the distribution of the logarithm of the resistances when $\sigma \rightarrow \infty$. Whatever the form of the initial distribution $\tilde{\Phi}(\ln r)$, the effective resistance of a large medium constituted by a large number of resistances converges to the median of $\tilde{\Phi}$ (see Fig. 20). In the case of binary mixtures of conducting $\left(r_{0}=0\right.$ or $\left.\ln r_{0}=-\infty\right)$ and insulating resistances $\left(r_{\infty}=\infty\right.$ or $\left.\ln r_{\infty}=\infty\right)$, the median of $\tilde{\Phi}(\ln r)$ is equal to $-\infty$ or else $+\infty$ depending on the probability $\left(0 \leq p_{0} \leq 1\right)$ to have conducting resistances. Indeed, if $p_{0}>0.5$, there are more conducting resistances than insulating resistances in the mixture and the median of $\tilde{\Phi}(\ln r)$ is $-\infty$. In that case, the effective resistance of the mixture is also $-\infty$. On the contrary, for $p_{0}<0.5$, the median of $\tilde{\Phi}$ is $+\infty$ and the corresponding effective resistance is $+\infty$. When $p_{0}=0.5$, changing only one resistance in the mixture (for example an insulating resistance becomes a conductive resistance) is sufficient to change the state of the mixture from insulating to conducting, even if the mixture is constituted by a very large number of resistances. This very sensitive transition from an insulating state to a conducting state is called percolation and, in that case $(2 \mathrm{D}, z=4)$, the percolation threshold is equal to 0.5 (see appendix B).

\subsection{Three-dimensional systems}

Here the results obtained previously for 2D systems are generalized for three-dimensional materials like powders. The effective resistance given by Eq. (35) is compared to the one calculated numerically thanks to the same kind of iteration process used to describe a $2 \mathrm{D}$ material (see appendix A.2).

\subsubsection{For not very wide distributions $(\sigma<1)$}

For 3D systems with $z=6$ and for not very wide distributions, Kirpatrick's equation can be approximated as (see appendix C)

$$
\delta=-\frac{\sigma^{2}}{6}
$$

where $\delta=\ln r_{\text {eff }}-\langle\ln r\rangle$. With the same approximation (order 2 on $\sigma$ ), Landau [27] remarked that this result is identical to that given by the following equation:

$$
r_{e f f}=\left\langle r^{-1 / 3}\right\rangle^{-3},
$$

showing that Eq. (38) is replaced by Eq. (43) for 3D systems. The advantage of such equation is the possible extension to imaginary impedances, giving access to the frequency dependence of the effective impedance [6]. However, De Wit [28] pushed the development up to the sixth order and showed differences at this level, depending on the shape of the distribution of resistance values.

The results from the iterative process are compared with Eqs. (35), (42) and (43) in Fig. 21 for three different distributions of resistances: log-normal law (plusses) and two skewed distributions with $S$ equal to one (squares)

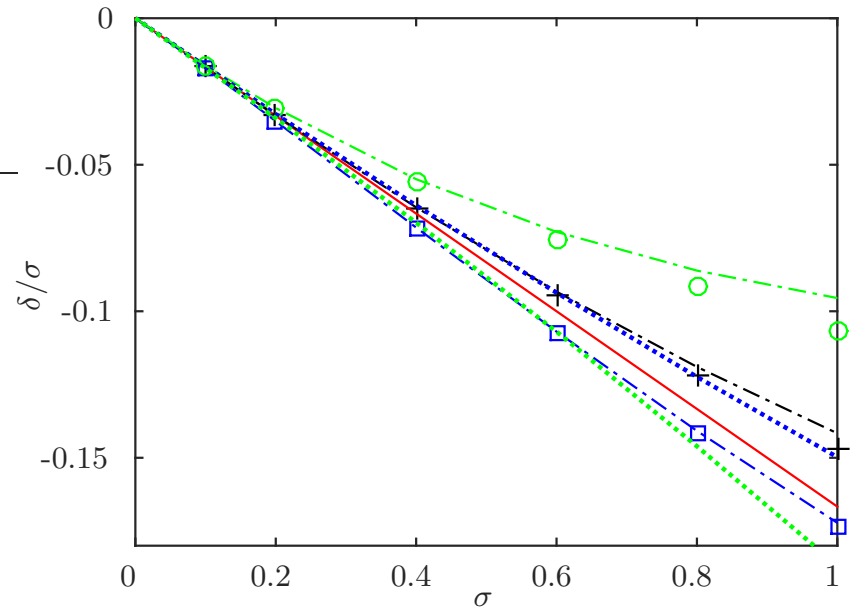

Fig. 21. Results from the iterative process and comparison with Eqs. (35), (42) and (43). Plusses: $\delta / \sigma$ obtained for a normal distribution $\Phi(u)$ or a log-normal distribution for $\phi(r)$. Squares: results for a skewed distribution with $S=1$ whatever $\sigma$. Circles: results for a skewed distribution with $S=-1$. Dot-dashed lines: Eq. (35), with $z=6$. Solid line: Eq. (42). Dotted lines: Eq. (43). The two extreme lines correspond to the skewed distribution of the resistances with $S=-1$.

and minus one (circles). These two skewed distributions are the ones obtained previously in the case of 2D systems. For $\sigma<1$, very few differences are observed between the Kirkpatrick's equation (35) and the effective resistance calculated numerically, whatever the value of the skewness chosen. Besides, the approximation (42) stays valid for $\sigma<0.2$ but greater differences are observed when $\sigma$ is greater than 0.2 . As for the more practical approximation given by Landau, Eq. (43) gives the same result than Eq. (42) for resistances distributed by a log-normal law, but, when $\sigma>0.2$, differences between Eq. (43) and the Kirkpatrick's equation are slightly larger than the ones observed between Eqs. (42) and (35).

\subsubsection{From very wide distributions to percolation theory}

Contrary to that observed for $2 \mathrm{D}$ systems, when $\sigma$ is larger than one, large differences are observed between the effective resistance calculated numerically and the one given by the Kirpatrick's equation both for a log-normal distribution and the two skewed distributions (see Fig. 22). When $\sigma$ tends to infinity, Eq. (35) with $z=6$ can be approximated by

$$
-\int_{-\infty}^{\ln r_{e f f}} \tilde{\Phi}(\ln r) \mathrm{d} \ln r+\frac{1}{2} \int_{\ln r_{e f f}}^{\infty} \tilde{\Phi}(\ln r) \mathrm{d} \ln r=0
$$

and hence:

$$
Q_{\text {eff }, \sigma \rightarrow \infty}^{\text {Kirkatrick }}=\frac{1}{3},
$$

where the quantile $Q_{e f f}=\int_{-\infty}^{\ln r_{e f f}} \tilde{\Phi}(\ln r) \mathrm{d} \ln r$ is the proportion of resistances smaller than the effective resistance. 


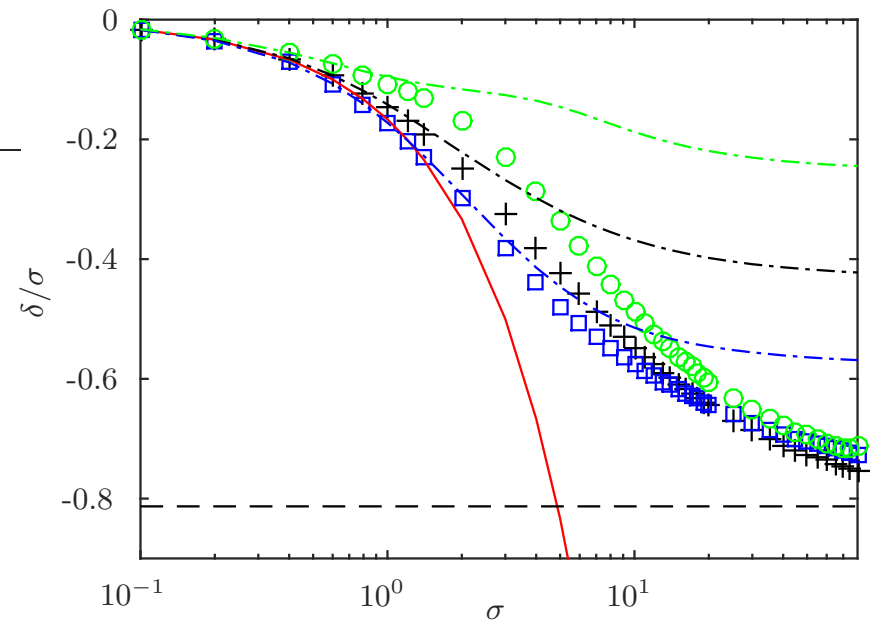

Fig. 22. $\delta / \sigma$ versus $\sigma$ (same symbols as Fig. 21). Solid line: Eq. (42). Dot-dashed lines: Eq. (35), with $z=6$. Horizontal line: Eq. (47).

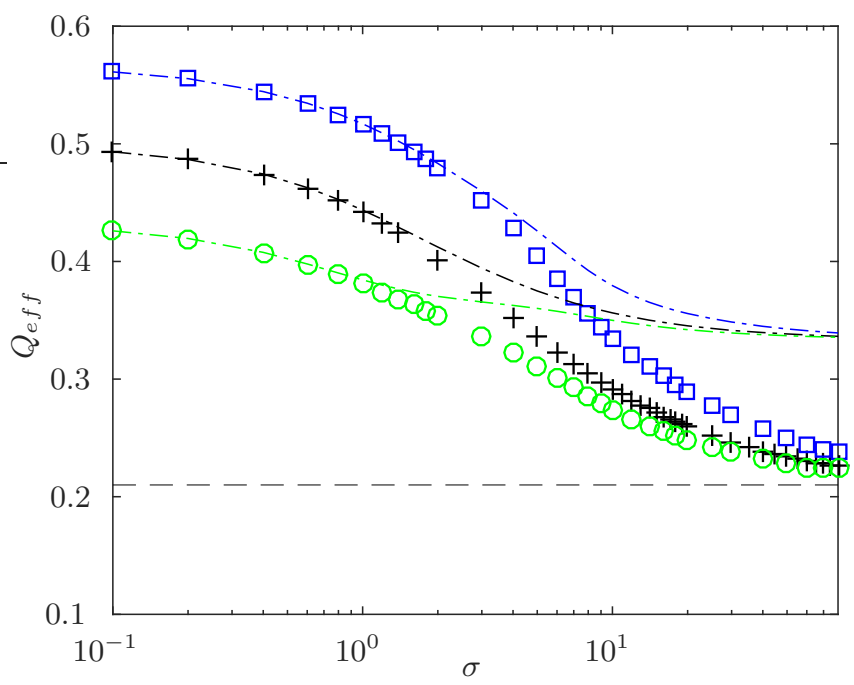

Fig. 23. The quantile $Q_{\text {eff }}$ versus $\sigma$. Same symbols as Figs. 21 and 22. For dot-dashed lines, the quantile is calculated using the effective resistance given by (35). Horizontal and dashed line: $Q_{e f f, \sigma \rightarrow \infty}=p_{c}=0.208$.

Figure 23 shows that, using the effective resistance given by the Kirkpatrick's equation, $Q_{\text {eff }}$ tends to $1 / 3$ whatever the skewness of the distribution of resistances (dot-dashed lines). But, the quantile calculated from the numerical results is quite different and tends to another value, close to 0.2 .

We can then focus on binary mixtures of conducting $\left(r_{0}=0\right.$ or $\left.\ln r_{0}=-\infty\right)$ and insulating resistances $\left(r_{\infty}=\infty\right.$ or $\left.\ln r_{\infty}=\infty\right)$. In that case, $\sigma=\infty$ and using Eq. (45), $Q_{\text {eff, } \sigma \rightarrow \infty}=1 / 3$, whereas $Q_{\text {eff }, \sigma \rightarrow \infty} \approx 0.2$ from the results we have obtained thanks to the iteration process. So, using the same argument presented previously for $2 \mathrm{D}$ systems, the effective medium theory $(35)$ gives $1 / 3$ for the percolation threshold $\left(p_{c}\right)$ whereas it is rather close to
0.2 adopting the iteration process. We have checked this result in appendix $\mathrm{B}$ and, for the $3 \mathrm{D}$ system, we have found

$$
Q_{e f f, \sigma \rightarrow \infty}=p_{c}=0.208 \pm 0.001 .
$$

Coming back to the effective resistance issue, Eq. (46) gives the value of $\ln r_{\text {eff }}$ whatever the form of the distribution but when $\sigma$ tends to infinity. For a log-normal distribution of the resistances, Eq. (46) yields to

$$
\left(\frac{\delta}{\sigma}\right)_{\sigma \rightarrow \infty}=-0.813
$$

For the two skewed distributions studied in this article ( $S=1$ and $S=-1$ ), equation (46) gives respectively $(\delta / \sigma)_{\sigma \rightarrow \infty}=-0.791$ and $(\delta / \sigma)_{\sigma \rightarrow \infty}=-0.777$, showing that the three curves $\delta / \sigma$ versus $\sigma$ in Fig. 22 are very close when $\sigma$ tends to infinity.

\subsection{Comparison 1D/2D/3D}

Here we summarize the results obtained in this section:

- The 3D case is the only one for which there is no equation to calculate directly the effective resistance from the distribution of the resistances. For 1D systems, $r_{\text {eff }}^{1 D}=\langle r\rangle$ whereas for 2D systems Kirkpatrick's equation (35) gives $r_{e f f}^{2 D}$. Besides, for $2 \mathrm{D}$ systems and for symmetrical distributions of the logarithm of the resistances, Eq. (35) implies that $\ln r_{\text {eff }}=\langle\ln r\rangle$ whatever the value of the standard deviation.

- For not very broad distributions that is for distributions with a small standard deviation (typically $\sigma<1$ but it depends on the precision needed for obtaining the effective resistance and also on the form of the distribution of the resistances), $\ln r_{\text {eff }}=\langle\ln r\rangle$ for $2 \mathrm{D}$ systems whereas $r_{\text {eff }}=\left\langle r^{-1 / 3}\right\rangle^{-3}$ for 3D systems.

- When the standard deviation of the distribution of resistances tends to infinity, the percolation theory gives the value of the effective resistance of a large medium using the following equation

$$
\int_{0}^{r_{e f f}} \phi(r) \mathrm{d} r=p_{c}
$$

where $p_{c}$ is the percolation threshold for a binary mixture of conducting and insulating resistances. $p_{c}$ is also equal to the quantile which is the proportion of resistances smaller than the effective resistance when considering a medium constituted by a large number of resistances distributed according to $\phi$. At two dimensions with $z=4, p_{c}=0.5$ and hence the effective resistance is the median of the distribution of the resistances. At three dimension and considering a network sketched in Fig. 26, $p_{c}=0.208$ and Eq. (48) gives the effective resistance depending on the distribution of the resistances.

Hence, whatever the dimension, when the size of the medium increases, its resistance converges to one limit 
called the "effective resistance" whose value depends both on the dimension and also on the form of the distribution of the resistances at small scale. To conclude on the effects of the dimension, we can also compare how "quickly" the resistance of the medium converges to the "effective resistance" as the size of the medium increases. Indeed, for a small size medium, the resistance depends on the sample chosen whereas, when the size is sufficiently large, the resistance becomes independent on the sample chosen. This has been clearly observed experimentally since the resistance of the chain of beads depends a lot on the beads chosen or even on the electrical contacts between the beads, but the standard deviation of the resistance measured from a large number of different chains of beads clearly decreases when the number of beads that constitute the chain increases (see Fig. 16). For the powder, the number of grains is large (more than 100000) and the measured resistance is very reproducible, depending only on the mechanical force and on the size of the sample. Thus, considering the three systems studied here (1D, 2D, 3D), we can compare directly the evolution of the standard deviation of the resistance as a function of the number of resistances being in the medium. For each system, we take the same distribution of resistances that is a log-normal law with the same standard deviation $\sigma_{0}$. Then, using the iteration process presented in appendix A, we calculate for each system the standard deviation of the new distribution of resistance at step 1 , then at step 2, etc... Thus, in Fig. 24 , the ratio between the standard deviation calculated at step $i$ and the initial one $\left(\sigma_{0}\right)$ is plotted against the number of components $\left(N_{c}\right)$ that constitute the medium. For the $1 \mathrm{D}$ system, $N_{c}=2^{i}$ whereas for the $2 \mathrm{D}$ and $3 \mathrm{D}$ systems, $N_{c}=2^{2 i}$ and $N_{c}=2^{3 i}$ respectively. Indeed, at each step, each piece of material is constituted by 4 smaller pieces of materials at 2D (Fig. 25) and 8 pieces of materials at 3D (Fig. 26).

For an initial distribution of resistances not very large i.e. $\sigma_{0}$ small (in Fig. 24 we take $\sigma_{0}=0.1$ ), $\sigma_{i}$ decreases with the number of components that constitute the medium at a similar rate whatever its dimension. Indeed, in appendix $\mathrm{D}$, we show that, for small $\sigma_{0}$, a very good approximation of $\sigma_{i} / \sigma_{0}$ is $2^{-i / 2}(1 \mathrm{D}), 2^{-i}(2 \mathrm{D})$ and $2^{-3 i / 2}$ (3D). Hence, $\sigma_{i} / \sigma_{0}$ is a power law of the number of the components

$$
\frac{\sigma_{i}}{\sigma_{0}}=\frac{1}{\sqrt{N_{c}}}
$$

For broader distributions of initial resistances and whatever the dimension of the system, the standard deviation $\sigma_{i}$ decreases less quickly with the number of components than for Eq. (49). However, for 2D and 3D systems, Fig. 24 shows that the ratio $\sigma_{i} / \sigma_{0}$ stays decreasing quickly with the number of resistances whereas, for the 1D system, $\sigma_{i} / \sigma_{0}$ decreases very slowly. For example, for a system with 10000 resistances, $\sigma_{i} / \sigma_{0}$ is lower than $5 \%$ in two or three dimensions whereas $\sigma_{i} / \sigma_{0} \approx 28 \%$ at $1 \mathrm{D}$. This result confirms that the powder can be seen as an effective medium: for all the samples of powder tested in this article, the number of grains is greater than 100000 and hence the corresponding measured resistance is very close

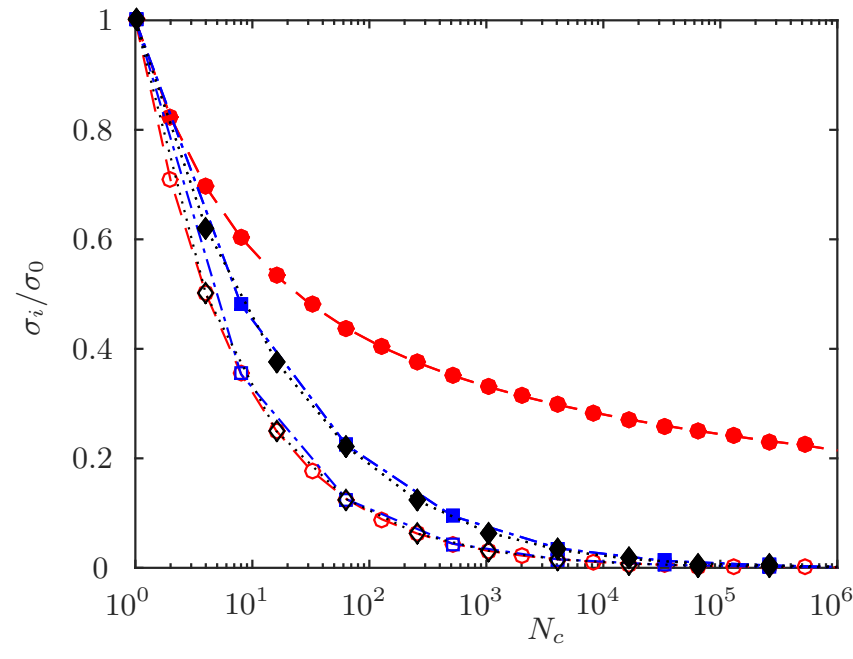

Fig. 24. Evolution of the standard deviation with the number of components that constitute the medium. Circles: 1D, $N_{c}=$ $2^{i}$. Diamonds: 2D, $N_{c}=2^{2 i}$. Squares: 3D, $N_{c}=2^{3 i} . \sigma_{0}$ is the standard deviation of the initial distribution of the resistances, open symbols: $\sigma_{0}=0.1$, plain symbols: $\sigma_{0}=10$.

to the effective resistance $\left(r_{\text {eff }}\right)$ of the powder. On the contrary, for the chain of beads, the measured resistance depends on the contacts between the beads and the resistance varies a lot even with the same beads and the same mechanical force. However, the effective resistance of the chain of beads can be calculated since, for a 1D system, $r_{e f f}=\langle r\rangle$.

\section{Conclusions}

We have measured the pressure dependence of the electrical resistance of one- and three-dimensional metallic granular media. Whatever the dimension of the granular medium, the resistance is found to be a power law of the pressure $\left(R \propto P^{-\alpha}\right)$ with an "anomalous" exponent $\alpha$ larger than the one expected either with elastic or plastic contact between the grains. The value of $\alpha$ is of course not "universal" and depends on the physical properties of both the grains and the oxide that covers the grains. But the experiments presented here on the chain of beads show that a large exponent $\alpha$ is also observed for a 1D granular system for which there is only one "force chain" and the stress is perfectly homogeneous since all the contacts between the grains undergo the same force. Besides, for the powders, the exponent for the capacitance is not larger than one $(C \propto P)$ showing that the pressure-dependent capacitance is well explained by a basic model (Eq. 10) without taking account the 3D structure of the force network. These results suggest that the strong dependence of the resistance on the force does not come from the nonhomogeneous distribution of the stresses in the 3D granular media, but it can be understood by means of a simple model of the electrical conduction between two metallic grains in contact. This model states that the electric current flowing from one grain to the other in contact does 
not pass uniformly in the oxide: it rather follows some conducting micro-channels. Due to strong inhomogeneities in the physical properties of the oxide (size and conductivity), the conductances of these micro-channels can be widely distributed following a very large distribution like a Pareto distribution. Then, the parameter $0<\mu<1$ of this Pareto distribution characterizes the variability of the properties of the micro-channels and controls directly the exponent $\alpha$ of the relation: $R \propto P^{-\alpha}\left(\alpha=\frac{2}{3 \mu}\right.$ in the elastic regime, $\alpha=\frac{1}{\mu}$ in the plastic regime).

The second main result of this work concerns the apparent contradiction arising from the measurements of the powder resistance. On the one hand, the pressuredependent resistance suggests that the individual contact resistance between the grains follows a very broad distribution, which should naively yields to a wide dispersion of the experimental results. On the other hand, packed powder samples behave well as an effective medium with very good reproducible conductivity measurements. Our renormalization approach solves this apparent contradiction. It shows that, in three dimensions, the distribution of resistances rapidly narrows when the size of the sample grows, explaining the reproducibility of the measurements. Further, it illustrates the crossover between two regimes, the narrow distribution regime, which extends up to standard deviations of the logarithm of resistances of order one, where the Landau's equation (43) holds, and the wide distribution limit, where the effective resistance of the sample is controlled by the percolation threshold.

\section{Acknowledgments}

The PCMS2I of the Ecole centrale de Lyon is gratefully acknowledge for providing computational resources.

\section{Author contribution statement}

CL and MC performed the experiments. BC and EF supervised the research work. MC drafted the manuscript and the figures. All authors reviewed the manuscript and gave final approval for publication.

\section{Appendix A Renormalization group method for calculating the effective resistance}

For a chain of random resistors, the corresponding effective resistance is given by the mean value of the resistance distribution. For 2D or 3D networks of resistors, Kirkpatrich's formula (35) gives an aproximation of the effective resistance but there is no exact equation for calculating $r_{\text {eff }}$. Here, the renormalization group method is used to calculate numerically the effective resistance at a given resistance distribution.

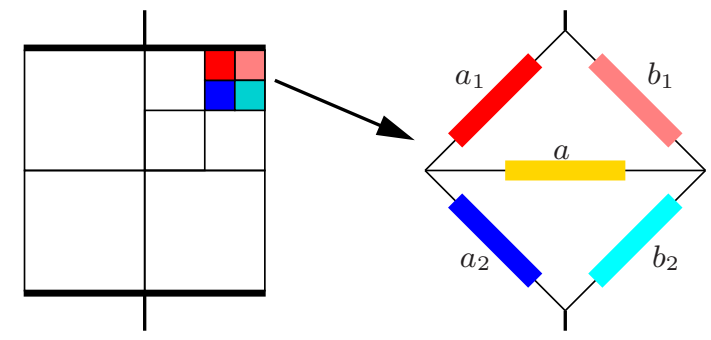

Fig. 25. Left: description of the iterative process. Each square piece of the material is divided in four smaller squares of half side length. Right: arrangement of the resistances for the iteration process. The four resistances $a_{1}, b_{1}, a_{2}$ and $b_{2}$ represent the resistances of the four sub-squares of side half of the square of material. The resistance $a$ represents the lateral conduction between these sub-squares.

\section{A.1 2D systems}

Instead of a lattice, we consider an inhomogeneous resistive material, and evaluate its effective resistivity by the following iterative process. Consider a square of this material, which we divide into four sub-squares, assumed homogeneous. The goal is to estimate the distribution of effective resistivity of the square, given the distribution of the resistances of the sub-squares. For this, we approximate this assembly of sub-squares with a block of five resistances $\left(a_{1}, a_{2}, b_{1}, b_{2}\right.$ and $\left.a\right)$, shaped as two triangles base to base (see Fig. 25). At a given scale, the following equation gives the electrical resistance $(r)$ of each square piece of the material as a function of these five resistances which are defined at smaller scale:

$$
r=\frac{a_{2} b_{2}\left(a_{1}+b_{1}\right)+a_{1} b_{1}\left(a_{2}+b_{2}\right)+a\left(a_{1}+a_{2}\right)\left(b_{1}+b_{2}\right)}{a\left(a_{1}+b_{1}+a_{2}+b_{2}\right)+\left(a_{1}+b_{1}\right)\left(a_{2}+b_{2}\right)}
$$

At each step, this process corresponds to an averaging of the resistances over five resistances reflecting that the resistance of the square is an averaging of the resistances of the corresponding sub-squares. We can also notice that if all the sub-squares have the same resistance $\left(a_{1}=a_{2}=\right.$ $\left.b_{1}=b_{2}=a\right)$, the larger square has also the same resistance, showing that the effective resistance is size-independent at two dimensions. For the chain of beads, the effective resistance is proportional to the length of the chain (or the number of contact bead/bead) whereas, at three dimensions, the effective resistance is proportional to the height of the sample and inversely proportional to its crosssection [Eq. (15)].

Hence, given the distribution of the resistances at very small scale $(\phi)$, one can calculate the distribution of the resistances at greater scales using at each step Eq. (A.1). For each initial distribution $\phi, 5^{11}$ values of resistance are chosen randomly following $\phi$. The first step consists to average these resistances using Eq. (A.1) in order to obtain $5^{10}$ new values of resistance characterizing the distribution $\phi_{1}$ at step one. Then, from this new set of resistances, $5^{9}$ new values of resistance are calculated in order 
to determine the distribution at step two $\left(\phi_{2}\right)$. The process is continued until step 11 for which there is only one calculated resistance. After each step, the obtained distribution is of course narrower than the previous one since it is an averaging process. So the standard deviation of the distribution of the resistances decreases as the number of steps increases and finally it tends to zero after 11 iterations whatever the initial standard deviation tested hereafter. We then assume that the resistance obtained after 11 steps is very close to the one we search that is the effective resistance of the material at large scale $\left(r_{e f f}\right)$.

\section{A.2 3D systems}

Here, a cube of the material is divided into eight sub-cubes and the goal is to estimate the distribution of resistance of the cube, given the distribution of the resistances of the sub-cubes (see Fig. 26). For this, we approximate this assembly of sub-cubes with a block of 12 resistances, shaped as two pyramids base to base. The eight resistances $a_{i}, b_{i}$, $c_{i}$ and $d_{i}(i=1$ or 2$)$ represent the resistances of the eight sub-cubes whereas the four resistances $(a, b, c$ and $d$ ) represent the lateral conduction between the sub-cubes. Equation (A.1) used for the 2D case needs to be generalized for this 3D structure. Indeed, the electrical resistance of the cube is a complex function $\left(\mathcal{G}_{3 D}\right)$ of these twelve resistances:

$$
r_{\text {cube }}=\mathcal{G}_{3 D}\left(a, b, c, d, a_{1}, b_{1}, c_{1}, d_{1}, a_{2}, b_{2}, c_{2}, d_{2}\right) .
$$

We have obtained $\mathcal{G}_{3 D}$ using Maple but it is not possible to write here this very long equation. Besides, if all of the subcubes have the same resistance (i.e. the twelve resistances are equal), the larger cube has a resistance twice smaller. This is in agreement with the fact that the resistance of a cube is inversely proportional to its size contrary to the square for which its resistance is size-independent. So, the resistance at step $i$ is defined as $r_{i}=2 \times \mathcal{G}_{3 D}$ in order to have $r_{i \rightarrow \infty}=r_{e f f}$, where $r_{e f f}$ is the effective resistance of the material.

At a given scale, using Eq. (A.2), we have calculated the resistance of each cube of material as a function of twelve resistances and all of them are defined at smaller scale. Hence, from the distribution of the resistances given at the smallest scale, we have obtained consecutively the distribution of the resistances at greater scale. After nine iterations, the standard deviation of the obtained distribution is very small and the value of the corresponding resistance is assumed to be the effective resistance of the material at large scale $\left(r_{e f f}\right)$.

\section{Appendix B Percolation thresholds for 2D and 3D lattices}

In the case of binary mixtures of conducting $\left(r_{0}=0\right.$ or $\left.\ln r_{0}=-\infty\right)$ and insulating resistances $\left(r_{\infty}=\infty\right.$ or $\ln r_{\infty}=\infty$ ), the percolation phenomenon is an insulating to conducting transition or else a conducting to insulating

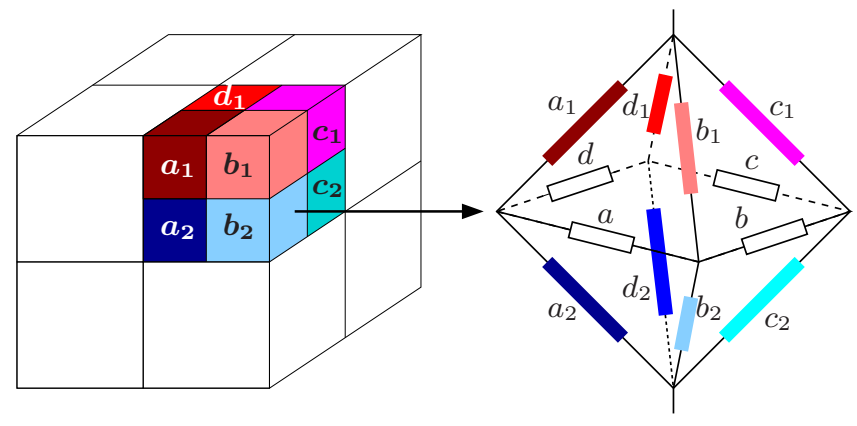

Fig. 26. Left: description of the iterative process. Each cube piece of the material is divided in eight smaller cubes of half side length. Right: arrangement of the resistances for the iteration process. The eight resistances $a_{1}, b_{1}, c_{1}, d_{1}$ and $a_{2}, b_{2}, c_{2}$, $d_{2}$ represent the resistances of the eight sub-cubes of side half of the cube of material. The resistances $a, b, c$ and $d$ represent the lateral conduction between these sub-cubes.

transition for the whole mixture depending only on the state of one resistance. This transition is observed when the proportion of conducting resistances in the mixture $\left(p_{0}\right)$ reaches a particular value $\left(p_{c}\right)$. This critical proportion of conducting resistances depends on the dimension and also on the geometrical characteristics of the resistor network. Here, using the iteration process described in Appendix A.1 and A.2, we calculate numerically $p_{c}$ for 2D and 3D networks that are described in Figs. 25 and 26 respectively.

At the smallest scale, there are two kind of resistances: the insulating ones $\left(r=r_{\infty}\right)$ and the conducting ones $(r=$ $\left.1 / r_{\infty}\right)$, with $\ln r_{\infty}=25$, and the proportion of conducting resistances is $p_{0}$. The first step consists to use respectively Eq. (A.1) (2D) or Eq. (A.2) (3D) in order to calculate the resistance of each block constituted by five (2D) or twelve (3D) initial resistances. Then, the distribution of the resistances at step one is obtained and the proportion of conducting resistances $\left(p_{1}\right)$ is evaluated. To calculate $p_{1}, \ln r=0$ is taken as the limit between a conducting resistance and an insulating one since the resistances at step one can take more than two values. The same process is used a second time in order to obtain the distribution of the resistances at step two and hence the proportion of conducting resistances $p_{2}$. And so on for simulating binary mixtures with a large number of initially resistances.

Figures 27 and 28 show the evolution of $p_{i}$ as a function of the initial proportion of conducting resistances $\left(p_{0}\right)$ after step 1,2, 3, 6 and 10 . For a binary mixture constituted of $5^{6}=15625$ resistances, except for $p_{0}$ close to $p_{c}$ with $p_{c}=0.5$ for the $2 \mathrm{D}$ system and $p_{c}=0.208 \pm 0.001$ (3D), $p_{6}$ is equal to 0 or 1 , depending on $p_{0}$. Thus, for binary mixtures constituted by a large number of resistances, the mixture is in an insulating state when $p_{0}<p_{c}$ whereas it becomes a conductor when $p_{0}>p_{c}$. When $p_{0}$ is close to $p_{c}$, the state of the mixture is very sensitive to the ratio between the numbers of conducting resistances and of insulating ones. 


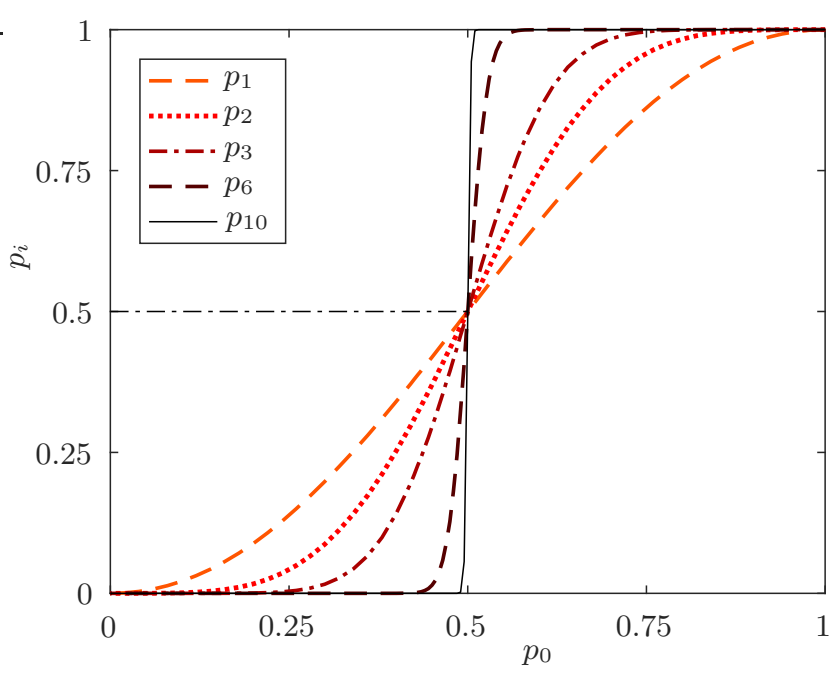

Fig. 27. Results for binary mixtures for the $2 \mathrm{D}$ system and using the iterative process. $p_{0}$ is the proportion of conducting resistances in the mixture. At step 10, $p_{10}$ is the corresponding proportion of conducting blocks and is very close to the one we search for a very large mixture.

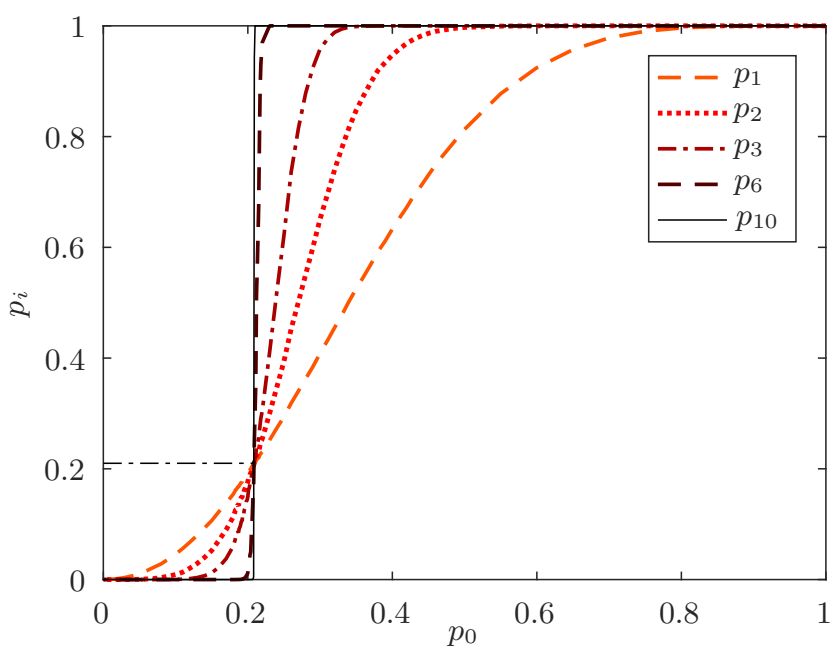

Fig. 28. Results for binary mixtures for the $3 \mathrm{D}$ system. At step $10, p_{10}=0$ when $p_{0}<p_{c}$ whereas $p_{10}=1$ when $p_{0}>p_{c}$ except for $p_{0}$ very close to $p_{c}$, with $p_{c} \approx 0.208$.

\section{Appendix C Approximation of Kirpatrick's equation for 3D systems}

Kirpatrick's equation (35) can be approximated for not very wide distributions $(\sigma<1)$ and for $z=6(z$ is the number of bonds meeting at each node of the network). First, Eq. (35) can be rewritten as

$$
\int_{-\infty}^{\infty} \frac{\exp (\delta-\sigma u)-1}{1+\frac{1}{3}[\exp (\delta-\sigma u)-1]} \Phi(u) \mathrm{d} u=0,
$$

with $u=(\ln r-\langle\ln r\rangle) / \sigma, \sigma^{2}=\left\langle(\ln r-\langle\ln r\rangle)^{2}\right\rangle$ and $\delta=$ $\ln r_{\text {eff }}-\langle\ln r\rangle$. Using the Taylor series expansion of the exponential function and omitting terms of order greater than two, Eq. (C.1) becomes $\delta^{2}+6 \delta+\sigma^{2}=0$ and when $\sigma$ is small, we obtain

$$
\delta=\ln r_{e f f}-\langle\ln r\rangle \approx-\frac{\sigma^{2}}{6} .
$$

Besides, using also the Taylor series expansion of the exponential function and then the one of the logarithm, we have

$$
\begin{aligned}
\ln \left(\left\langle r^{-\frac{1}{3}}\right\rangle^{-3}\right) & =-3 \ln \left[\int_{-\infty}^{\infty} \exp \left(-\frac{\sigma u+\langle\ln r\rangle}{3}\right) \Phi(u) \mathrm{d} u\right] \\
& \approx\langle\ln r\rangle-3 \ln \left[1+\frac{1}{2}\left(\frac{\sigma}{3}\right)^{2}\right] \\
& \approx\langle\ln r\rangle-\frac{\sigma^{2}}{6}
\end{aligned}
$$

From Eqs. (C.2) and (C.3), we conclude

$$
\ln r_{e f f}=\ln \left(\left\langle r^{-\frac{1}{3}}\right\rangle^{-3}\right) .
$$

\section{Appendix D Evolution of $\sigma$ with the number of resistances for not very wide distributions ( $\sigma_{0}$ small)}

\section{D.1 1D systems}

The iteration process has been used in this article for the $2 \mathrm{D}$ and $3 \mathrm{D}$ systems but this process can also be used for the chain of beads in order to evaluate the evolution of $\sigma$ as a function of the number of resistances that constitute the chain of beads. At each step $i+1$, the resistance $r_{i+1}$ is the mean of two resistances ( $a$ and $b$ ) defined at step $i$ : $r_{i+1}=(a+b) / 2$. This latter equation can be rewritten as

$$
\exp \left(\left\langle u_{i+1}\right\rangle+\delta u_{i+1}\right)=\exp \left(\left\langle u_{i}\right\rangle\right) \frac{\exp \left(\delta u_{a}\right)+\exp \left(\delta u_{b}\right)}{2}
$$

where $\left\langle u_{i}\right\rangle$ and $\left\langle u_{i+1}\right\rangle$ are respectively the mean of the logarithm of the resistances defined at step $i$ and $i+1$. Taking the logarithm of Eq. (D.1) and using the Taylor series expression of the exponential and logarithm functions, we obtain

$\left\langle u_{i+1}\right\rangle+\delta u_{i+1}=\left\langle u_{i}\right\rangle+\frac{\delta u_{a}+\delta u_{b}}{2}+\frac{\delta u_{a}^{2}+\delta u_{b}^{2}}{8}-\frac{\delta u_{a} \delta u_{b}}{4}$.

Taking the mean of (D.2) and using $\left\langle\delta u_{a}\right\rangle=\left\langle\delta u_{b}\right\rangle=0$ and $\left\langle\delta u_{a} \delta u_{b}\right\rangle=0$, it yields

$$
\left\langle u_{i+1}\right\rangle=\left\langle u_{i}\right\rangle+\frac{\left\langle\delta u_{i}^{2}\right\rangle}{4},
$$

and

$$
\left\langle\delta u_{i+1}^{2}\right\rangle=\frac{\left\langle\delta u_{i}^{2}\right\rangle}{2} .
$$

From Eq. (D.4) we obtain

$$
\frac{\sigma_{i}}{\sigma_{0}}=\sqrt{\frac{\left\langle\delta u_{i}^{2}\right\rangle}{\left\langle\delta u_{0}^{2}\right\rangle}}=\left(\frac{1}{2}\right)^{i / 2}
$$


Then, using Eqs (D.2) and (D.3), we have

$$
\left\langle u_{i+1}\right\rangle+\frac{\left\langle\delta u_{i+1}^{2}\right\rangle}{2}=\left\langle u_{i}\right\rangle+\frac{\left\langle\delta u_{i}^{2}\right\rangle}{2} .
$$

Finally, when $i$ tends to infinity, this latter equation gives

$$
\delta=\left\langle u_{\infty}\right\rangle-\left\langle u_{0}\right\rangle=\frac{\sigma_{0}^{2}}{2}
$$

which is an approximation of Eq. (34) when $\sigma_{0}$ is small.

\section{D.2 2D systems}

The calculus to obtain $\sigma_{i} / \sigma_{0}$ is much more complex at $2 \mathrm{D}$ than the one given previously for the chain of beads since the resistance at step $i+1\left[r_{i+1}=\exp \left(\left\langle u_{i+1}\right\rangle+\delta u_{i+1}\right)\right]$ is a function of five resistances: $a=\exp \left(\left\langle u_{i}\right\rangle+\delta u_{a}\right), a_{j}=$ $\exp \left(\left\langle u_{i}\right\rangle+\delta u_{a_{j}}\right)$ and $b_{j}=\exp \left(\left\langle u_{i}\right\rangle+\delta u_{b_{j}}\right)$, with $j=1$ or 2 . Eq. (A.1) gives $r_{i+1}$ as a function of these five resistances. Similarly to what was done for the 1D case, using the Taylor series expression of the exponential and the logarithm functions, we can obtain $\left\langle u_{i+1}\right\rangle+\delta u_{i+1}-\left\langle u_{i}\right\rangle$ as a power series of the five variables $\delta u_{a}, \delta u_{a_{j}}$ and $\delta u_{b_{j}}$. Taking the mean of this equation and saying that $\left\langle\delta u_{a_{j}}\right\rangle=$ $\left\langle\delta u_{b_{j}}\right\rangle=\left\langle\delta u_{a_{j}} \delta u_{b_{l}}\right\rangle=\left\langle\delta u_{a_{j}} \delta u_{b_{l}}^{2}\right\rangle=0$ whatever $j$ and $l$, and $\left\langle\delta u_{a_{j}} \delta u_{a_{l}}\right\rangle=\left\langle\delta u_{a_{j}} \delta u_{a_{l}}^{2}\right\rangle=0$ for $j \neq l$, it yields

$$
\left\langle u_{i+1}\right\rangle=\left\langle u_{i}\right\rangle-\frac{5}{64}\left\langle\delta u_{i}^{3}\right\rangle
$$

At 2D, the first non-zero term in Eq. (D.8) is the third moment of $\delta u_{i}$ and not the standard deviation $\sigma_{i}$. The standard deviation and the third moment of $\delta u_{i+1}$ can also be calculated:

$$
\left\langle\delta u_{i+1}^{2}\right\rangle=\frac{\left\langle\delta u_{i}^{2}\right\rangle}{4} \text { and }\left\langle\delta u_{i+1}^{3}\right\rangle=\frac{\left\langle\delta u_{i}^{3}\right\rangle}{16} .
$$

From Eqs. (D.9) we obtain

$$
\frac{\sigma_{i}}{\sigma_{0}}=\left(\frac{1}{2}\right)^{i} \text { and } \frac{\left\langle\delta u_{i}^{3}\right\rangle}{\left\langle\delta u_{i}^{2}\right\rangle^{3 / 2}}=S\left(\frac{1}{2}\right)^{\frac{3 i}{2}}
$$

where $S$ is the skewness of the initial distribution of resistances. Eq. (D.10) means that the distribution of $\ln r$ rapidly narrows and, even if the original distribution is skewed, it rapidly goes to a symmetrical distribution. Then, using Eqs. (D.8) and (D.10), we have

$$
\left\langle u_{i+1}\right\rangle-\frac{\left\langle\delta u_{i+1}^{3}\right\rangle}{12}=\left\langle u_{i}\right\rangle-\frac{\left\langle\delta u_{i}^{3}\right\rangle}{12} .
$$

Finally, when $i$ tends to infinity, this latter equation gives

$$
\delta=\left\langle u_{\infty}\right\rangle-\left\langle u_{0}\right\rangle=-\frac{S}{12} \sigma_{0}^{3},
$$

which is an approximation of Eq. (39) when $\sigma_{0}$ is small.

\section{D.3 3D systems}

For the 3D system (Fig. 26), the function $\mathcal{G}_{3 D}$ (A.2) gives $r_{i+1}=\exp \left(\left\langle u_{i+1}\right\rangle+\delta u_{i+1}\right)$ as a function of twelve resistances: $\zeta_{j}=\exp \left(\left\langle u_{i}\right\rangle+\delta u_{\zeta_{j}}\right)$, with $\zeta=a, b, c$ or $d$, and $j=$ 1,2 or \{\} . Using the Taylor series expression of the exponential and the logarithm functions, and with the help of Maple, we can obtain $\left\langle u_{i+1}\right\rangle+\delta u_{i+1}-\left\langle u_{i}\right\rangle$ as a power series of the twelve variables $\delta u_{\zeta_{j}}$. Taking the mean of this equation and saying that $\left\langle\delta u_{\zeta_{j}}\right\rangle=\left\langle\delta u_{\zeta_{j}} \delta u_{\xi_{l}}\right\rangle=\left\langle\delta u_{\zeta_{j}} \delta u_{\xi_{l}}^{2}\right\rangle=0$ if $\zeta \neq \xi$ or else $j \neq l$, it yields

$$
\left\langle u_{i+1}\right\rangle=\left\langle u_{i}\right\rangle+\frac{7}{48}\left\langle\delta u_{i}^{2}\right\rangle-\frac{35}{576}\left\langle\delta u_{i}^{3}\right\rangle .
$$

The standard deviation and the third moment of $\delta u_{i+1}$ can also be calculated

$$
\begin{aligned}
\left\langle\delta u_{i+1}^{2}\right\rangle & =\frac{\left\langle\delta u_{i}^{2}\right\rangle}{8}+\frac{7}{192}\left\langle\delta u_{i}^{3}\right\rangle, \\
\left\langle\delta u_{i+1}^{3}\right\rangle & =\frac{\left\langle\delta u_{i}^{3}\right\rangle}{64}
\end{aligned}
$$

From Eqs. (D.14) and (D.15) we obtain

$$
\begin{aligned}
\frac{\sigma_{i}}{\sigma_{0}} & \approx\left(\frac{1}{2}\right)^{\frac{3 i}{2}}\left(1-\frac{S}{3} \sigma_{0}\right), \\
\frac{\left\langle\delta u_{i}^{3}\right\rangle}{\left\langle\delta u_{i}^{2}\right\rangle^{3 / 2}} & =S\left(\frac{1}{2}\right)^{\frac{3 i}{2}},
\end{aligned}
$$

where $S$ is the skewness of the initial distribution of resistances. Then, using Eqs. (D.13), (D.16) and (D.17) we have

$$
\left\langle u_{i+1}\right\rangle-\frac{\left\langle\delta u_{i+1}^{2}\right\rangle}{6}-\frac{\left\langle\delta u_{i+1}^{3}\right\rangle}{18}=\left\langle u_{i}\right\rangle-\frac{\left\langle\delta u_{i}^{2}\right\rangle}{6}-\frac{\left\langle\delta u_{i}^{3}\right\rangle}{18} .
$$

Finally, when $i$ tends to infinity, this latter equation gives

$$
\delta=\left\langle u_{\infty}\right\rangle-\left\langle u_{0}\right\rangle=-\frac{1}{6} \sigma_{0}^{2}-\frac{S}{18} \sigma_{0}^{3},
$$

and when $\sigma_{0}$ is very small, Eq. (D.19) gives Eq. (42).

\section{References}

1. R. W. Wilson. Proc. Roy. Soc. Lond. B, 68:625, 1955.

2. T. Travers, M. Ammi, D. Bideau, A. Gervois, J.-C. Messager, and J.-P. Troadec. Europhys. Lett., 4:329, 1987.

3. M. Ammi, T. Travers, D. Bideau, A. Gervois, J.-C. Messager, and J.-P. Troadec. J. Phys., 49:221, 1988.

4. D. Vandembroucq, A. C. Boccara, and S. Roux. J. Phys. III, 7:303, 1997.

5. M. Creyssels, S. Dorbolo, A. Merlen, C. Laroche, B. Castaing, and E. Falcon. Eur. Phys. J. E, 23:255, 2007.

6. M. Creyssels, E. Falcon, and B. Castaing. Phys. Rev. B, 77:075135, 2008.

7. H. A. Janssen. Z. Ver. Dtsch. Ing., 39:1045, 1895.

8. K. L. Johnson. Contact Mechanics. Cambridge Univ. Press, Cambridge, 1985. 
9. R. Holm. Electric Contacts. Springer Verlag, Berlin, 4th edition, 2000.

10. F. P. Bowden. Proc. Roy. Soc. Lond. A, 169:391, 1939.

11. K. L. Euler. J. Power Sources, 3:117, 1978.

12. M. Creyssels, E. Falcon, and B. Castaing. AIP Conf. Proc., 1145:123, 2009.

13. CU 006025. Goodfellow product catalogue, 2004.

14. J. D. Bernal and J. Mason. Nature, 188:910, 1960.

15. G. D. Scott. Nature, 188:908, 1960.

16. G. D. Scott and D. M. Kilgour. Br. J. Appl. Phys. (J. Phys. D.), 2:863, 1969.

17. D. Bideau M. Ammi and J. P. Troadec. J. Phys. D: Appl. Phys., 20:424, 1987.

18. E. Falcon, B. Castaing, and C. Laroche. Europhys. Lett., 65:186, 2004

19. E. Falcon, B. Castaing, and M. Creyssels. Eur. Phys. J.

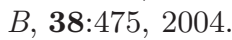

20. E. Branly. C. R. Acad. Sci. Paris, 111:785, 1890.

21. N. Nunõ, R. Groppetti, and N. Senin. Clin. Biomech., 21:956, 2006.

22. L. Vanel, Ph. Claudin, J.-Ph. Bouchaud, M. E. Cates, E. Clément, and J. P. Wittmer. Phys. Rev. Lett., 84:1439, 2000.

23. B. V. Gnedenko and A. N. Kolmogorov. Limit Distributions for Sums of Independent Random Variables. Addison-Wesley, London, 1954.

24. J. P. Bouchaud and A. Georges. Phys. Rep., 195:127, 1990.

25. S. Kirkpatrick. Phys. Rev. Lett., 27:1722, 1971.

26. S. Kirkpatrick. Rev. Mod. Phys., 45:574, 1973.

27. L. D. Landau and E. M. Lifshitz. Electrodynamics of Continuous Media., volume 8. Pergamon Press, 1960.

28. A. De Wit. Phys. Fluids, 7:2553, 1995. 